Homology, Homotopy and Applications, vol.20(2), 2018, pp.315-339

\title{
ON MATRIX TODA BRACKETS IN BAUES-WIRSCHING COHOMOLOGY
}

\author{
YASUHIRO MOMOSE AND KENICHIROU SHINKAI
}

\author{
(communicated by J. Daniel Christensen)
}

\begin{abstract}
Hardie, Kamps and Marcum have considered the matrix Toda brackets introduced by Barratt in the category of topological spaces from a 2-categorical point of view. Baues and Dreckmann have shown that a class in the third Baues-Wirsching cohomology of a small category $\mathcal{C}$ governs every classical Toda bracket if the bracket is defined with a Toda category in $\mathcal{C}$. Our aim is to generalize such a relationship to that between the class in the cohomology and matrix Toda brackets in a 2category. Moreover, the non-triviality of the third cohomology is discussed via computation of a matrix Toda bracket in the category of cochain complexes on an additive category.
\end{abstract}

\section{Introduction}

The secondary operations due to Toda [21], the Toda brackets, play a crucial role in the computation of the homotopy groups of spheres. The construction of the Toda bracket is applicable in a triangulated category $[\mathbf{1 1}, \mathbf{1 6}]$. Thus one might expect that such a secondary operation gives us new insights into consideration of an appropriate Abelian category, a triangulated category and a 2-category; see $[\mathbf{3}, \mathbf{5}, \mathbf{8}, \mathbf{1 3}, \mathbf{1 4}, \mathbf{1 5}$, 20]. Baues and Wirsching have introduced cohomology of a small category, the BauesWirsching cohomology, with interpretation of the first and second cohomology; see [9]. Baues and Dreckmann [8] have shown that the third Baues-Wirsching cohomology of a small category $\mathcal{C}$ classifies linear track extensions over $\mathcal{C}$. Moreover, a particular class, the so-called universal Toda bracket is defined in the third one. If the category $\mathcal{C}$ is a subcategory of the category of topological spaces, then it is shown that the universal Toda bracket governs every Toda bracket if $\mathcal{C}$ contains the Toda category, which defines the bracket; see [8].

Generalizing the classical Toda brackets, matrix Toda brackets have been introduced by Barratt [2]. Subsequently, Hardie, Kamps and Marcum [13] have developed a categorical approach to such brackets in a 2-category. The results together with those mentioned above motivate us to investigate the generalized brackets with the cohomology of a small category.

Received March 29, 2017, revised August 25, 2017, March 9, 2018; published on June 27, 2018. 2010 Mathematics Subject Classification: 18D05, 18E30, 55U35.

Key words and phrases: track category, linear track extension, universal Toda bracket, triangulated category.

Article available at http://dx.doi.org/10.4310/HHA.2018.v20.n2.a16

Copyright (C) 2018, International Press. Permission to copy for private use granted. 
In this manuscript, we show that the same class as the universal Toda bracket in the third Baues-Wirsching cohomology of a small category also governs matrix Toda brackets in the sense of Hardie, Kamps and Marcum if the bracket is decomposed into two Toda brackets; see Theorem 2.6. Moreover, using the description of the classical Toda bracket in a triangulated category due to Heller, we examine the non-triviality of a matrix Toda bracket defined in a 2-category of cochain complexes. Indeed, it is possible to represent a matrix Toda bracket with a classical one in an algebraic triangulated category; see Theorem 2.14 for more details. The formula is reminiscent of the original definition of the matrix Toda bracket due to Barratt; see also [19].

\section{Acknowledgments}

The authors would like to express their deep thanks to Katsuhiko Kuribayashi for his many valuable comments on this article and his encouragement throughout our research for this work. They thank the referee for careful reading, thoughtful suggestions and valuable proposals to revise a previous version of this paper.

\section{The main theorems}

In this section, our main theorems are described. For a small category $\mathcal{C}$, we define the category $\mathcal{F}(\mathcal{C})$ of factorizations in $\mathcal{C}$ as follows. The objects are the morphisms in $\mathcal{C}$ and a morphism from $\alpha$ to $\beta$ is a pair $(u, v)$ of morphisms in $\mathcal{C}$ such that $\beta=u \circ \alpha \circ v$ :

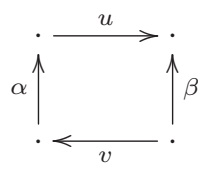

The composite of maps $(u, v): \alpha \rightarrow \beta$ and $\left(u^{\prime}, v^{\prime}\right): \beta \rightarrow \gamma$ is defined by $\left(u^{\prime}, v^{\prime}\right) \circ$ $(u, v)=\left(u^{\prime} \circ u, v \circ v^{\prime}\right)$. Observe that, for an object $\varphi: y \rightarrow x$ of $\mathcal{F}(\mathcal{C})$, a morphism $i d_{\varphi}=\left(i d_{x}, i d_{y}\right)$ is the identity on $\varphi$. By definition, a natural system $D$ on a small category $\mathcal{C}$ is a covariant functor from $\mathcal{F}(\mathcal{C})$ to the category of Abelian groups. We may write $D_{\alpha}$ for $D(\alpha)$, where $\alpha \in \operatorname{ob}(\mathcal{F}(\mathcal{C}))$.

We recall the definition of a 2-category. A category $\mathcal{G}$ is a 2-category if the following conditions are satisfied:

(i) For objects $X, Y \in \mathrm{ob}(\mathcal{G})$, the hom-set $\operatorname{Hom}_{\mathcal{G}}(X, Y)$ constitutes a small category $\mathcal{G}(X, Y)$ with ob $\mathcal{G}(X, Y)=\operatorname{Hom}_{\mathcal{G}}(X, Y)$.

(ii) For $X, Y, Z \in \mathrm{ob}(\mathcal{G})$, the composite of morphisms in $\mathcal{G}$ defines a functor $\circ: \mathcal{G}(Y, Z) \times \mathcal{G}(X, Y) \rightarrow \mathcal{G}(X, Z)$ with $1_{1_{Y}} \circ-=1_{\mathcal{G}(X, Y)}$ and $-\circ 1_{1_{Y}}=1_{\mathcal{G}(Y, Z)}$.

A morphism from $f: X \rightarrow Y$ to $g: X \rightarrow Y$ denoted $F: f \Rightarrow g$ in the category $\mathcal{G}(X, Y)$ is called a 2-morphism.

An object 0 in a 2-category is a 0 -object if $\mathcal{G}(0, X)$ and $\mathcal{G}(X, 0)$ are trivial categories for any objects $X \in \mathrm{ob}(\mathcal{G})$.

Two 1-morphisms $f, g: A \rightarrow B$ of $\mathcal{G}$ are homotopic if there exists an invertible 2-morphism $F: f \Rightarrow g$. We write $f \simeq g$ if $f$ and $g$ are homotopic.

As usual, the homotopy category $H \mathcal{G}$ of a 2-category $\mathcal{G}$ is the category with the same class of objects as that in $\mathcal{G}$ and the hom-set $\operatorname{Hom}_{H \mathcal{G}}(A, B)$ for objects $A$ and 
$B$ in $H \mathcal{G}$ is defined to be the quotient $\operatorname{Hom}_{\mathcal{G}}(A, B) / \simeq$ by the homotopy relation $\simeq$. In what follows, $\mathcal{G}$ denotes a 2 -category with a 0 -object unless otherwise specified.

In order to describe our main theorem (Theorem 2.6 below), we further need terminology on 2-morphisms.

Definition 2.1. Let $S: u \Rightarrow v$ and $T: r \Rightarrow s$ be 2-morphisms, where $u, v, r$, and $s$ are morphisms with same source and target. We say that $S$ and $T$ are conjugate and write $S\langle$ conj $\rangle T$ if there exists invertible morphisms $H: v \Rightarrow s$ and $K: u \Rightarrow r$ such that $H+S=T+K$.

Definition 2.2. Let $f, g: A \rightarrow B$ be morphisms and $F: f \Rightarrow g$ a 2-morphism in $\mathcal{G}$. A set $N(F)$ is defined to be the set $\left\{G: 0_{B}^{A} \Rightarrow 0_{B}^{A} \mid G\langle\right.$ conj $\left.\rangle F\right\}$.

In what follows, we write $\mathcal{A}_{X Y}$ for $N\left(1_{0}: 0 \Rightarrow 0: X \rightarrow Y\right)$. We need more structure on the set $\mathcal{A}_{X Y}$ in order to relate matrix Toda brackets with the cohomology mentioned in the Introduction. The set $\mathcal{A}_{X Y}$ is automatically a group; see [13, Proposition 2.4]. Moreover, we assume that the group $\mathcal{A}_{X Y}$ is Abelian for any $X$ and $Y$ in $H \mathcal{G}$. For any morphisms $b: B \rightarrow C$ in $\mathcal{G}$, the maps $b_{*}: \mathcal{A}_{A B} \rightarrow \mathcal{A}_{A C}$ and $b^{*}: \mathcal{A}_{C A} \rightarrow \mathcal{A}_{B A}$ which are induced by the horizontal composition in $\mathcal{G}$ are well-defined homomorphisms by the interchange law. Observe that we have a functor $\mathcal{A}_{-,-}: H \mathcal{G} \times H \mathcal{G} \rightarrow A b$ to the category of Abelian groups.

Consider a commutative diagram



in $H \mathcal{G}$. Then we can define a matrix Toda bracket

$$
\left\{\begin{array}{ll}
b & g \\
a & f
\end{array}, w\right\}
$$

in the sense of Hardie, Kamps and Marcum; see Section 3 for more details. Let $h$ and $k$ be 1 -morphisms of a 2 -category $\mathcal{G}$. For a subset $\mathcal{C}$ of $\mathcal{G}(h, h)$ and an invertible 2-morphism $T: k \Rightarrow h$, let $\mathcal{C}^{T}$ denote the subset $\{-T+\xi+T \mid \xi \in \mathcal{C}\}$ of $\mathcal{G}(k, k)$; see $\left[\mathbf{1 3},(6.5)\right.$ Notation]. Then, we see that the matrix Toda bracket $\left\{\begin{array}{l}b, g \\ a\end{array}, w\right\}$ is in $\mathcal{A}_{W X} /\left(b_{*} \mathcal{A}_{W B}+\mathcal{D}+a_{*} \mathcal{A}_{W A}\right)$, where $\mathcal{D}$ is the submodule $(\mathcal{A}(a f) \circ w)^{a H}$ of $\mathcal{A}_{W X}$, where $\mathcal{A}(a f)=\{F: a f \Rightarrow a f \mid F:$ invertible $\}$. Moreover, if $a f \simeq 0 \simeq b g$, then $\left\{\begin{array}{l}b, g \\ a,\end{array}, w\right\}$ is in $\mathcal{A}_{W X} /\left(b_{*} \mathcal{A}_{W B}+w^{*} \mathcal{A}_{C X}+a_{*} \mathcal{A}_{W A}\right)$; see [13, Corollary 7.3]. In fact, the result $\left[\mathbf{1 3}\right.$, Proposition 6.9 (c)] yields that $\mathcal{D}=w^{*} \mathcal{A}_{C X}$.

Definition 2.3. Let $A b$ be the category of Abelian groups. A functor $D^{\mathcal{A}}: \mathcal{F}(H \mathcal{G}) \rightarrow$ $A b$ is defined by $D_{[f]}^{\mathcal{A}}:=\mathcal{A}_{s(f) t(f)}=N\left(1_{0}: 0 \Rightarrow 0: s(f) \rightarrow t(f)\right)$ and $D^{\mathcal{A}}(u, v)(G):=$ $1_{u} \circ G \circ 1_{v}=v^{*} u_{*} G$ for $G \in D_{[f]}^{\mathcal{A}}$ and $(u, v):[f] \rightarrow[g]$, where $\circ$ denotes the composition in $H \mathcal{G}$. 
Let $\mathcal{G}$ be a track category; see Definition 3.2 below. We consider a linear track extension of the form $D^{\mathcal{A}} \stackrel{+}{\longrightarrow} \mathcal{G}_{2} \longrightarrow \mathcal{G}_{1} \stackrel{p}{\longrightarrow} H \mathcal{G}$ in the sense of Baues and Dreckmann; see Definition 3.4. Then, we have the universal Toda bracket $\langle H \mathcal{G}\rangle$ in the Baues-Wirsching cohomology $H^{3}\left(H \mathcal{G}, D^{\mathcal{A}}\right)$ with the coefficients in the natural system $D^{\mathcal{A}}$. Here, $H \mathcal{G}$ is small; see Definition 3.6. We observe that one of important data which defines a linear track extension is a set of isomorphisms $\sigma_{f}: D_{[f]}^{\mathcal{A}} \rightarrow \mathcal{G}(f, f)$ determined by 1-morphisms $f$ of $\mathcal{G}$. Such a set of isomorphisms is called the action of $D^{\mathcal{A}}$ to $\mathcal{G}$.

Track categories appear naturally in homotopy theory. Indeed, applying the fundamental groupoid functor to each mapping space in a topologically enriched category yields a track category; see [17]. The discussion in [10, Introduction] as well as [12, Section 8.I,8, III.1] gives a simplicial version of the construction above. Moreover, a cofibration category in the sense of Baues gives rise to a track category; see [6, Proposition II.5.6 and Corollary]. From a category equipped with a suitable cylinder functor, one can form a track category as described in [17, Theorem IV.1.11].

We here mention that the Baues-Wirsching cohomology $H^{*}(\mathcal{C}, D)$ of a small category $\mathcal{C}$ can be normalized with an ideal $S$ of the category $\mathcal{C}$. We denote it by $H_{S}^{*}(\mathcal{C}, D)$; see Section 4.

Definition 2.4. Let $M T$ be the category generated by the directed graph in displayed diagram, modulo the relation $\tilde{a} \circ \tilde{f}=\tilde{b} \circ \tilde{g}$.

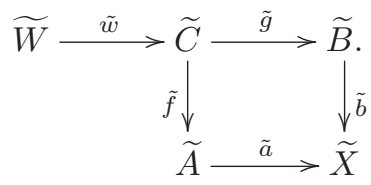

This category MT is called the matrix Toda category.

Definition 2.5. Let $\mathcal{C}$ be a category with zero morphisms. For a functor $F: M T \rightarrow \mathcal{C}$ which satisfies $F(f w)=0, F(g w)=0$, the functor $F$ is called a matrix Toda diagram in $\mathcal{C}$. We also call $F(M T)$ a matrix Toda diagram in $\mathcal{C}$.

For the category $M T$, we have a functor $\varphi: M T \rightarrow H \mathcal{G}$ with $\varphi(\tilde{\eta})=\eta$ for $\eta \in$ $\{w, a, b, f, g\}$; that is, the diagram (2.1) is regarded as a matrix Toda diagram. Then, the functor $\varphi$ induces a homomorphism $\varphi^{*}: H_{O(H \mathcal{G})}^{3}\left(H \mathcal{G}, D^{\mathcal{A}}\right) \rightarrow H_{S}^{3}\left(M T, \varphi^{*} D^{\mathcal{A}}\right)$. Here, $S=\{\tilde{f} \tilde{w}, \tilde{g} \tilde{w}, \tilde{b} \tilde{g} \tilde{w}\}$. Moreover, we have an isomorphism $\tilde{h}: H^{3}\left(H \mathcal{G}, D^{\mathcal{A}}\right) \rightarrow$ $H_{O(H \mathcal{G})}^{3}\left(H \mathcal{G}, D^{\mathcal{A}}\right)$; see Section 4 .

We are ready to describe our main theorem.

Theorem 2.6. Let $\mathcal{G}$ be a track category with $H \mathcal{G}$ small, which satisfies Hypothesis I; see Section 3. If af $\simeq 0 \simeq b g$, then there exists an isomorphism

$$
\alpha: H_{S}^{3}\left(M T, \varphi^{*} D^{\mathcal{A}}\right) \stackrel{\cong}{\longrightarrow} \mathcal{A}_{W X} /\left(b_{*} \mathcal{A}_{W B}+w^{*} \mathcal{A}_{C X}+a_{*} \mathcal{A}_{W A}\right)
$$


such that the composite defined by the diagram

$$
\begin{aligned}
& H_{O(H \mathcal{G})}^{3}\left(H \mathcal{G}, D^{\mathcal{A}}\right) \longrightarrow \varphi_{S}^{*}\left(M T, \varphi^{*} D^{\mathcal{A}}\right) \\
& \underset{h \uparrow}{H^{3}\left(H \mathcal{G}, D^{\mathcal{A}}\right) \stackrel{\left\{\begin{array}{ll}
b & g \\
a & f
\end{array}\right\}^{*},}{\longrightarrow} \mathcal{A}_{W X} /\left(b_{*} \mathcal{A}_{W B}+w^{*} \mathcal{A}_{C X}+a_{*} \mathcal{A}_{W A}\right)}
\end{aligned}
$$

sends the universal Toda bracket $\langle H \mathcal{G}\rangle$ to the matrix Toda bracket $\left\{\begin{array}{ll}b & g \\ a & f\end{array}, w\right\}$ defined by the diagram (2.1).

We recall a linear track extension; see Definition 3.4. Then we have the following corollary.

Corollary 2.7. Let $\mathcal{C}$ be a small subcategory of $H \mathcal{G}$ which has a matrix Toda diagram with a non-zero bracket. Then $C$ admits a non-trivial linear track extension by the natural system $D^{\mathcal{A}}$ mentioned in Theorem 2.6.

In fact, for the inclusion functor $\iota: \mathcal{C} \rightarrow H \mathcal{G}$, the morphism $\left\{\begin{array}{ll}b & g \\ a & f\end{array}, w\right\}^{*}$ in Theorem 2.6 factors through the restriction $\iota^{*}: H^{3}\left(H \mathcal{G}, D^{\mathcal{A}}\right) \rightarrow H^{3}\left(\mathcal{C}, \iota^{*} D^{\mathcal{A}}\right)$, which ensures $0 \neq \iota^{*}\langle H \mathcal{G}\rangle \in H^{3}\left(\mathcal{C}, \iota^{*} D^{\mathcal{A}}\right)$.

If $A$ in the diagram (2.1) is the 0 -object, then we have a diagram

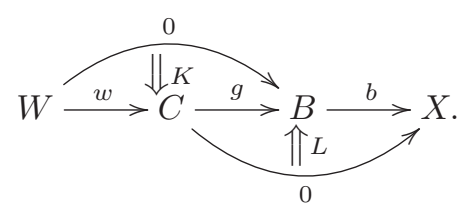

Therefore, the matrix Toda bracket $\left\{\begin{array}{ll}b & g \\ 0 & 0\end{array}, w\right\}$ is nothing but the classical Toda bracket $\{b, g, w\}$ defined by $\{-b K+L w \mid K: 0 \Rightarrow g w, L: 0 \Rightarrow b g, K, L:$ invertible $\}$. We observe that $\{b, g, w\}$ is in the coset $\mathcal{A}_{W X} /\left(b_{*} \mathcal{A}_{W B}+w^{*} \mathcal{A}_{C X}\right)$; see $[\mathbf{1 3}$, Proposition 8.2].

Corollary 2.8. One has $\left\{\begin{array}{ll}b & g \\ 0 & 0\end{array}, w\right\}^{*}(\langle H \mathcal{G}\rangle)=\left\{\begin{array}{lll}b & g & w\end{array}\right\}$.

In what follows, we may write $\{b g w\}^{*}$ for $\left\{\begin{array}{ll}b & g \\ 0 & 0\end{array}, w\right\}^{*}$.

Remark 2.9. We have $\left\{\begin{array}{ll}b & g \\ a & f\end{array}, w\right\}^{*}(\langle H \mathcal{G}\rangle)=\left\{\begin{array}{ll}b & g \\ a & f\end{array}, w\right\}=\{b g w\}-\{a f w\}=(\pi \circ$ $\left(\left\{b g w^{*}-\{a f w\}^{*}\right)\right)(\langle H \mathcal{G}\rangle)$ in $\mathcal{A}_{W X} /\left(b_{*} \mathcal{A}_{W B}+w^{*} \mathcal{A}_{C X}+a_{*} \mathcal{A}_{W A}\right)$, where $\pi$ is the projection map from $\mathcal{A}_{W X} /\left(b_{*} \mathcal{A}_{W B}+w^{*} \mathcal{A}_{C X}\right) \oplus \mathcal{A}_{W X} /\left(w^{*} \mathcal{A}_{C X}+a_{*} \mathcal{A}_{W A}\right)$ to $\mathcal{A}_{W X} /\left(b_{*} \mathcal{A}_{W B}+w^{*} \mathcal{A}_{C X}+a_{*} \mathcal{A}_{W A}\right) ;$ see Lemma 4.7 below.

We here recall a result in $[\mathbf{8}]$ on the classical Toda bracket. Let Top be the category of based topological spaces with the based homotopy relation $\simeq_{*}$. Assume 
that the 2-category $\mathcal{G}$ in our setting is a subcategory of based coHspaces whose 1-morphism are continuous maps and whose 2-hom-set of $\operatorname{Top}(X, Y)$ is defined by $\operatorname{Hom}_{T o p(X, Y)}(f, g)=\{H: X \times I \rightarrow Y\} / \simeq_{*}$ for 1-morphisms $f, g: X \rightarrow Y$. Then we see that

$$
\mathcal{A}_{A B}=N\left(1_{0}: 0_{B}^{A} \Rightarrow 0_{B}^{A}\right)=\left\{G: 0_{B}^{A} \Rightarrow 0_{B}^{A} \mid G\langle\operatorname{conj}\rangle 1_{0}\right\} \cong[\Sigma A, B]
$$

as a set and that Hypothesis I is satisfied for the 2-category $\mathcal{G}$. Observe that the based homotopy set $[\Sigma A, B]$ is an Abelian group whose addition is defined with the suspension structure of the domain as usual. Therefore, in view of Corollary 2.8, Theorem 2.6 is regarded as a generalization of the result [8, Theorem 3.3]. It is important to remark that the ideal $S^{\prime}$ used in [8, Theorem 3.3] does not coincide with $S$ in Theorem 2.6. In fact, $S=\{f w, g w, b g w\}$ and $S^{\prime}=\{b g, g w, b g w\}$. Hence, Corollary 2.8 gives another description of classical Toda brackets, which is stated in terms of matrix Toda brackets.

Under the same assumption as in Theorem 2.6, we have propositions. These propositions follow from [13, Theorems 5.4 and 5.5] and Theorem 2.6.

Proposition 2.10. Let $x: X \rightarrow Y$ be a morphism. Given a diagram of the form (2.1) in $H \mathcal{G}$, let $x_{*}: \mathcal{A}_{W X} /\left(b_{*} \mathcal{A}_{W B}+\mathcal{D}+a_{*} \mathcal{A}_{W A}\right) \rightarrow \mathcal{A}_{W Y} /\left((x b)_{*} \mathcal{A}_{W B}+\mathcal{A}_{C Y} \circ w+\right.$ $\left.(x a)_{*} \mathcal{A}_{W A}\right)$ be the homomorphism induced by $x$, where $\mathcal{D}=(\mathcal{A}(a f) \circ w)^{a H}$. Suppose further that $x a f=0=x b g$ in a commutative diagram

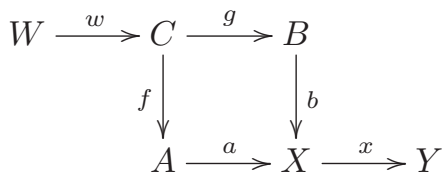

in $H \mathcal{G}$. Then, there holds the equality

$$
x_{*}\left\{\begin{array}{ll}
b & g \\
a & f
\end{array}, w\right\}=\left\{\begin{array}{ll}
x b & g \\
x a & f
\end{array}, w\right\}^{*}(\langle H \mathcal{G}\rangle)
$$

in $\mathcal{A}_{W X} /\left((x b)_{*} \mathcal{A}_{W B}+\mathcal{A}_{C Y} \circ w+(x a)_{*} \mathcal{A}_{W A}\right)$.


inition of the matrix Toda bracket, there exists an element $\theta$ in $\mathcal{A}_{W X}$ such that $\left\{\begin{array}{ll}b & g \\ a & f\end{array}, w\right\}=[\theta]$. It follows that $x_{*}\left\{\begin{array}{ll}b & g \\ a & f\end{array}, w\right\}=[x \circ \theta]$. The result $[\mathbf{1 3}$, Theorem 5.4 $]$ implies that $x \circ\left\{\begin{array}{ll}b & g \\ a & f\end{array}, w\right\} \subset\left\{\begin{array}{ll}x b & g \\ x a & f\end{array}, w\right\}$. Then we see that $x \circ \theta \in x \circ\left\{\begin{array}{ll}b & g \\ a & f\end{array}, w\right\}$ and hence $[x \circ \theta]=\left\{\begin{array}{ll}x b & g \\ x a & f\end{array}, w\right\}$ in the quotient mentioned in the assertion. This completes the proof.

Proposition 2.11. Given a diagram of the form (2.1) in $H \mathcal{G}$, let $\pi: \mathcal{A}_{Z X} /\left(b_{*} \mathcal{A}_{Z B}+\right.$ $\left.\mathcal{D}+a_{*} \mathcal{A}_{Z A}\right) \rightarrow \mathcal{A}_{Z X} /\left(b_{*} \mathcal{A}_{Z B}+\mathcal{A}_{W X} \circ \delta+a_{*} \mathcal{A}_{Z A}\right)$ be the projection, where $\delta: Z \rightarrow$ 
$W$ is a morphism and $\mathcal{D}=(\mathcal{A}(a f) \circ w \delta)^{a H}$. Suppose that af $w=0=$ bgw in a commutative diagram

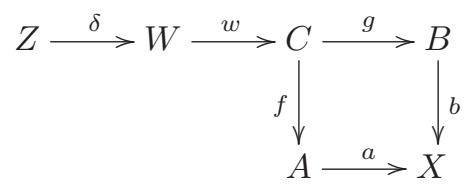

in HG. Then, one has

$$
\pi\left\{\begin{array}{ll}
b & g \\
a & f
\end{array}, w \delta\right\}=\left\{\begin{array}{ll}
b & g w \\
a & f w
\end{array}, \delta\right\}^{*}(\langle H \mathcal{G}\rangle)
$$

in $\mathcal{A}_{Z X} /\left(b_{*} \mathcal{A}_{Z B}+\mathcal{A}_{W X} \circ \delta+a_{*} \mathcal{A}_{Z A}\right)$.

Proof. Theorem 2.6 enables us to deduce that $\left\{\begin{array}{ll}b & g w \\ a & f w\end{array}, \delta\right\}^{*}(\langle H \mathcal{G}\rangle)=\left\{\begin{array}{ll}b & g w \\ a & f w\end{array}, \delta\right\}$. By the definition of the matrix Toda bracket, there exists an element $\theta$ in $\mathcal{A}_{Z X}$ such that $\left\{\begin{array}{ll}b & g \\ a & f\end{array}, w \delta\right\}=[\theta]$. By $\left[\mathbf{1 3}\right.$, Theorem 5.5], we see that $\left\{\begin{array}{ll}b & g \\ a & f\end{array}, w \delta\right\} \subset\left\{\begin{array}{ll}b & g w \\ a & f w\end{array}, \delta\right\}$. Since $\theta$ is in $\left\{\begin{array}{ll}b & g \\ a & f\end{array}, w \delta\right\}$, it follows that $[\theta]=\left\{\begin{array}{ll}b, & g w \\ a, & f w\end{array}, \delta\right\}$ in the quotient. We have the result.

In describing Theorem 2.6, we use a matrix Toda category $M T$ but not a Toda diagram in [8]. Therefore, the maps $\pi$ and $x_{*}$ in Propositions 2.10 and 2.11 are defined naturally. An advantage of the propositions above is that the non-triviality of the matrix Toda brackets follows from that of the image by the homomorphisms of the universal Toda bracket $\langle H \mathcal{G}\rangle$.

Another main theorem asserts that a matrix Toda bracket is represented by the classical one in an appropriate category. In order to describe such a result, we consider the category of cochain complexes $C h(\mathcal{B})$ on an additive category $\mathcal{B}$.

For any objects $X$ and $Y$ in $C h(\mathcal{B})$, the hom-set $C h(\mathcal{B})(X, Y)$ admits a category structure. In fact, its objects are cochain maps from $X$ to $Y$ and the hom-set is a set of linear maps of degree -1 defined by

$$
\operatorname{Hom}_{C h(\mathcal{B})(X, Y)}(f, g):=\left\{h: X \rightarrow Y \mid f-g=d_{Y} h+h d_{X}\right\} / \sim .
$$

Moreover, for linear maps $h, k: X \rightarrow Y$ of degree -1 , by definition $h \sim k$ if and only if there exists a linear map $u: X \rightarrow Y$ of degree -2 such that $h-k=d_{Y} u-u d_{X}$.

A vertical composite

$$
+: \operatorname{Hom}_{C h(\mathcal{B})(X, Y)}(g, h) \times \operatorname{Hom}_{C h(\mathcal{B})(X, Y)}(f, g) \rightarrow \operatorname{Hom}_{C h(\mathcal{B})(X, Y)}(f, h)
$$

is defined by $+\left(h_{1}, h_{2}\right)=h_{1}+h_{2}$. Moreover, the composite

$$
\text { ○: } C h(\mathcal{B})(Y, Z) \times C h(\mathcal{B})(X, Y) \rightarrow C h(\mathcal{B})(X, Z)
$$

in the category $C h(\mathcal{B})$ gives rise to a functor whose behavior in the hom-sets

$$
\circ: \operatorname{Hom}_{C h(\mathcal{B})(Y, Z)}\left(f^{\prime}, g^{\prime}\right) \times \operatorname{Hom}_{C h(\mathcal{B})(X, Y)}(f, g) \rightarrow \operatorname{Hom}_{C h(\mathcal{B})(X, Z)}\left(f^{\prime} \circ f, g^{\prime} \circ g\right)
$$


is defined by $k \circ h=f^{\prime} k+h g$ for $(k, h) \in \operatorname{Hom}_{C h(\mathcal{B})(Y, Z)}\left(f^{\prime}, g^{\prime}\right) \times \operatorname{Hom}_{C h(\mathcal{B})(X, Y)}(f, g)$.

The homotopy category $H(C h(\mathcal{B}))$ admits the triangulated category structure whose distinguished triangles are constructed by the mapping cone and suspension functors; see [1, Theorem 2.3.1].

More generally, we recall the definition of an algebraic triangulated category.

Definition 2.12 ([18, 3.2]). An exact functor $T \rightarrow \mathcal{U}$ between triangulated categories is a pair $(v, \eta)$ consisting of a functor $v: \mathcal{T} \rightarrow \mathcal{U}$ and natural isomorphism $\eta: v \circ \Sigma_{\mathcal{T}} \rightarrow \Sigma_{\mathcal{U}} \circ v$ such that for every exact triangle $X \stackrel{\alpha}{\rightarrow} Y \stackrel{\beta}{\rightarrow} Z \stackrel{\gamma}{\rightarrow} \Sigma X$ in $\mathcal{T}$ the triangle

$$
v X \stackrel{v \alpha}{\longrightarrow} v Y \stackrel{v \beta}{\longrightarrow} v Z \stackrel{\eta X \circ v \gamma}{\longrightarrow} \Sigma(v X)
$$

is exact in $\mathcal{U}$.

Definition $2.13([\mathbf{1 8}, 7.5])$. A triangulated category $\mathcal{T}$ is called algebraic if there exists a fully faithful exact functor $\vartheta: \mathcal{T} \rightarrow H(C h(\mathcal{B}))$ with a natural isomorphism $\eta: \vartheta \circ \Sigma \rightarrow \Sigma \circ \vartheta$ for some additive category $\mathcal{B}$.

A matrix Toda diagram (2.1) in a triangulated category $\mathcal{T}$ gives rise to a Toda diagram of the form

$$
W \stackrel{w}{\longrightarrow} C \stackrel{-f \vee g}{\longrightarrow} A \oplus B \stackrel{\nabla(a, b)}{\longrightarrow} X .
$$

In fact, we see that $(-f \vee g) w=0$ and $\nabla(a, b)(-f \vee g)=0$ in $\mathcal{T}$. Let $\{\varphi, \psi, \eta\}$ denote the classical Toda bracket for $X \stackrel{\eta}{\rightarrow} Y \stackrel{\psi}{\rightarrow} Z \stackrel{\varphi}{\rightarrow} W$ in $\mathcal{T}$ defined by Heller [16, Section 13]. Then we have the following theorem.

Theorem 2.14. For a matrix Toda diagram (2.1) in an algebraic triangulated category $\mathcal{T}$ with a fully faithful exact functor $(\vartheta, \eta)$. Via $\vartheta$, one has

$$
\left\{\begin{array}{ll}
b, & g \\
a, & f
\end{array}\right\}=\{\nabla(a, b),-f \vee g, w\},
$$

that is

$$
\left\{\begin{array}{ll}
\vartheta b, & \vartheta g \\
\vartheta a, & \vartheta f
\end{array} \quad \vartheta w\right\}=\vartheta\{\nabla(a, b),-f \vee g, w\} \circ \eta_{W}^{-1}
$$

The rest of this paper is organized as follows. Section 3 gives a brief review of the matrix Toda bracket introduced by Hardie, Kamps and Marcum. Moreover, we recall the definition of the Baues-Wirsching cohomology of a small category with coefficients in a natural system. After recalling a linear track extension over a small category, the universal Toda bracket is defined. In Section 4, we prove Theorem 2.6 defining the map $\left\{\begin{array}{ll}b & g \\ a & f\end{array}, w\right\}^{*}$ explicitly. Section 5 proves Theorem 2.14 and gives a computational example of a matrix Toda bracket. 


\section{Brief recollection on matrix Toda brackets and the univer- sal Toda bracket}

We begin by considering a diagram

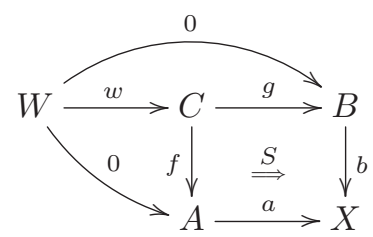

in a 2-category $\mathcal{G}$, where $S: a f \Rightarrow b g$ is a 2-morphism. Then, we define a set $\sigma(S, w)$ by $\sigma(S, w):=\{-b K+S w+a H \mid H: 0 \Rightarrow f w, K: 0 \Rightarrow g w, H$ and $K$ are invertible $\}$.

We define the matrix Toda bracket $\left\{\begin{array}{ll}b & g \\ a & f\end{array}, w\right\}$ to be the union $\bigcup_{S \in Z} \sigma(S, w)$, where $Z=\{S \mid S: a f \Rightarrow b g$ is invertible $\}$.

Remark 3.1. For a 2-morphism $S: a f \Rightarrow b g$ and 1-morphism $w: W \rightarrow \mathrm{C}$, we see that $\sigma(S, w) \subset N(S w)$.

Let $\theta=-b K+S w+a H$ in $N\left(1_{a f w}\right)=\mathcal{A}_{W X}$; see [13, Proposition (2.4)]. Suppose that $a f \simeq 0 \simeq b g$ and $\mathcal{A}_{W X}$ is an Abelian group. Then, the matrix Toda bracket $\left\{\begin{array}{ll}b & g \\ a & f\end{array}, w\right\}$ coincides with the $\operatorname{coset}\left(\theta+b \circ N\left(1_{g w}\right)+N(S) \circ w+a \circ N\left(1_{f w}\right)\right)$. We observe that $a \simeq a^{\prime}, b \simeq b^{\prime}, f \simeq f^{\prime}, g \simeq g^{\prime}$ and $w \simeq w^{\prime}$ then $\left\{\begin{array}{ll}b & g \\ a & f\end{array}, w\right\}=\left\{\begin{array}{ll}b^{\prime} & g^{\prime} \\ a^{\prime}, & f^{\prime},\end{array}, w^{\prime}\right\}$ as a coset; see [13, Proposition (5.3)].

In general, $k \simeq l$ then $N\left(1_{k}\right)=N\left(1_{l}\right)$; see [13, Proposition (2.2)]. Then, we see that $\left\{\begin{array}{ll}b & g \\ a & f\end{array}, w\right\}=[\theta]$ is in $\mathcal{A}_{W X} /\left(b_{*} \mathcal{A}_{W B}+w^{*} \mathcal{A}_{C X}+a_{*} \mathcal{A}_{W A}\right)$.

Let $\mathcal{C}$ be a small category and $D$ a natural system. For $\alpha, u \in \operatorname{mor}(\mathcal{C})$ with $s(u)=$ $t(\alpha)$, we write $u_{*}$ for the homomorphism $D\left(u, \operatorname{id}_{s(\alpha)}\right): D_{\alpha} \rightarrow D_{u \circ \alpha}$. Similarly, the homomorphism $D\left(\operatorname{id}_{t(\alpha)}, v\right): D_{\alpha} \rightarrow D_{\alpha \circ v}$ is written as $v^{*}$, where $v \in \operatorname{mor}(\mathcal{C})$ with $t(v)=s(\alpha)$.

We here recall the definition of the $n$th Baues-Wirsching cohomology of $\mathcal{C}$ with coefficients in $D$. For $n \geqslant 1$, let $N_{n}(\mathcal{C})$ be the set of $n$-simplices of the nerve of $\mathcal{C}$; that is, $N_{n}(\mathcal{C})=\left\{\lambda=\left(\lambda_{1}, \lambda_{2}, \ldots, \lambda_{n}\right) \mid X_{0} \stackrel{\lambda_{1}}{\leftarrow} X_{1} \stackrel{\lambda_{2}}{\leftarrow} X_{2} \leftarrow \cdots \stackrel{\lambda_{n}}{\leftarrow} X_{n}\right\}$. Let $F^{n}$ be an Abelian group defined by

$$
F^{n}=F^{n}(\mathcal{C}, D):=\left\{c: N_{n}(\mathcal{C}) \rightarrow \cup_{g \in \operatorname{mor}(\mathcal{C})} D_{g} \mid c\left(\lambda_{1}, \ldots, \lambda_{n}\right) \in D_{\lambda_{1} \circ \cdots \circ \lambda_{n}}\right\} .
$$

The boundary operator $\delta^{n}: F^{n-1} \rightarrow F^{n}$ is defined by

$$
\begin{aligned}
\left(\delta^{n}(c)\right)(\lambda)= & \lambda_{1 *} c\left(\lambda_{2}, \ldots, \lambda_{n}\right) \\
& +\sum_{i=1}^{n-1}(-1)^{i} c\left(\lambda_{1}, \ldots, \lambda_{i} \circ \lambda_{i+1}, \ldots, \lambda_{n}\right)+(-1)^{n} \lambda_{n}^{*} c\left(\lambda_{1}, \ldots, \lambda_{n-1}\right),
\end{aligned}
$$

for $\lambda=\left(\lambda_{1}, \ldots, \lambda_{n}\right) \in N_{n}(\mathcal{C})$ and $c \in F^{n-1}$. The cohomology $H^{n}\left(F^{*}(\mathcal{C}, D)\right)$ of the 
complex $F^{*}(\mathcal{C}, D):=\left\{F^{n}(\mathcal{C}, D), \delta^{n}\right\}_{n \in \mathbb{Z}}$ is called the $n$th Baues-Wirsching cohomology, denoted $H^{n}(\mathcal{C}, D)$.

Definition 3.2. A track category $\mathcal{T}$ is a 2-category enriched in groupoids. More precisely, $\mathcal{T}$ is category consisting of following data:

(i) For objects $A$ and $B$ of $\mathcal{T}$, the set $\mathcal{T}(A, B)$ of 1 -morphisms is a groupoid.

(ii) The composite $*: \mathcal{T}(A, B) \times \mathcal{T}\left(A^{\prime}, A\right) \rightarrow \mathcal{T}\left(A^{\prime}, B\right)$ is a functor for $A^{\prime}, A, B \in$ $\mathrm{ob}(\mathcal{T})$.

We may write $\mathcal{T}_{1}$ and $\mathcal{T}_{2}$ for the underlying 1-category of a track category and the sets of 2-morphisms, respectively.

Remark 3.3. (i) For $f, f^{\prime} \in \operatorname{Hom}_{\mathcal{T}_{1}}(A, B)=\operatorname{ob} \mathcal{T}(A, B), H \in \operatorname{Hom}_{\mathcal{T}(A, B)}\left(f, f^{\prime}\right)$ and $G \in \operatorname{Hom}_{\mathcal{T}\left(A^{\prime}, A\right)}\left(g, g^{\prime}\right)$, the function $*$ carries the pair of tracks $(H, G)$ to a track


cal composite + : Hom $\operatorname{To}(A, B)\left(f^{\prime}, f^{\prime \prime}\right) \times \operatorname{Hom}_{\mathcal{T}(A, B)}\left(f, f^{\prime}\right) \rightarrow \operatorname{Hom}_{\mathcal{T}(A, B)}\left(f, f^{\prime \prime}\right)$. Observe that the functoriality of $*$ gives rise to equalities $H * G=g^{\prime *} H+f_{*} G$ $=f_{*}^{\prime} G+g^{*} H$, where $f_{*} G:=1_{f} * G$ and $g^{*} H:=H * 1_{g}$.

(ii) $\left(1_{A}\right)_{*} G=G,\left(1_{A}\right)^{*} H=H$.

(iii) The operation $*$ in (i) is associative.

Definition 3.4 ([8, Definition (4.3)]). Let $\mathcal{C}$ be a category and $D$ a natural system on $\mathcal{C}$, namely a functor $D: \mathcal{F C} \rightarrow A b$. A linear track extension $\mathcal{E}$ of $\mathcal{C}$ by $D$ consists of a track category $\mathcal{T}$, a functor $p: \mathcal{T}_{1} \rightarrow \mathcal{C}$ and an action $\sigma$ of $D$ on $\mathcal{T}$, which is a set $\sigma=\left\{\sigma_{f}: D_{p(f)} \rightarrow \mathcal{T}(f, f) \mid \sigma_{f}\right.$ is an isomorphism of groups for $\left.f \in \operatorname{mor}\left(\mathcal{T}_{1}\right)\right\}$. Moreover, the following conditions are required:

(i) $p$ is identity on $\mathrm{ob}(\mathcal{C})$ and full, moreover, $p$ satisfies $p(f)=p(g) \Leftrightarrow f \simeq g$.

(ii) For $H \in \mathcal{T}(f, h)$ and $\alpha \in D_{p(f)}, \sigma_{h}(\alpha)+H=H+\sigma_{f}(\alpha)$.

(iii) For $\alpha, \beta \in D_{p(f)}$ and $f, g \in \operatorname{mor}\left(\mathcal{T}_{1}\right)$, one has $g^{*} \sigma_{f}(\alpha)=\sigma_{f g}\left(g^{*} \alpha\right)$ and $f_{*} \sigma_{g}(\beta)=$ $\sigma_{f g}\left(f_{*} \beta\right)$.

Following [8], we may write $D \stackrel{+}{\longrightarrow} \mathcal{T}_{2} \Longrightarrow \mathcal{T}_{1} \stackrel{p}{\longrightarrow} \mathcal{C}$ for the linear track extension $\mathcal{E}$ mentioned above, see also $[4$, Section 1$]$ for the notation.

Let $\mathcal{E}$ be a linear track extension of a small category $\mathcal{C}$ by $D$ and let $\tau: \operatorname{mor} \mathcal{C} \rightarrow$ mor $\mathcal{T}_{1}$ and $H: N_{2} \mathcal{C} \rightarrow \bigcup_{f, g \in \operatorname{mor}\left(\mathcal{T}_{1}\right)} \mathcal{T}(f, g)$ be functions with $p \circ \tau=1$ and $H(f, g) \in$ $\mathcal{T}(\tau f \circ \tau g, \tau(f g))$. We define a cochain $C_{\mathcal{E}}(\tau, H): N_{3}(\mathcal{C}) \rightarrow \bigcup_{f \in \operatorname{mor}(\mathcal{C})} D_{f}$ by

$$
C_{\mathcal{E}}(\tau, H)(f, g, h):=\sigma_{\tau(f g h)}^{-1}(\Delta),
$$

with the track $\Delta=-H(f, g h)-(\tau f)_{*} H(g, h)+(\tau h)^{*} H(f, g)+H(f g, h)$. See the diagram below. Observe that $\Delta$ belongs to $T(\tau(f g h), \tau(f g h))$ and $\sigma_{\tau(f g h)}^{-1}$ is an isomor- 
phism from $\mathcal{T}(\tau(f g h), \tau(f g h))$ to $D(p \tau(f g h))=D(f g h)$.



Let $F^{n}$ denote the Abelian group $F^{n}(\mathcal{C}, D)$. For the boundary operator $\delta^{4}: F^{3} \rightarrow$ $F^{4}$, we see that $\delta^{4}(C(\tau, H))=0$. Observe that the cocycle $C(\tau, H)$ does depend on the choice of $\tau$ and $H$; its cohomology class $[C(\tau, H)]$ dose not; see [8, (A.1) Lemma (c)].

Remark 3.5. Suppose that $\mathcal{T}$ has a 0 -object, then we can choose $\tau$ and $H$ so that $\tau(0)=0$ and $H(f, 0)=H(0, f)=1_{0}$. Indeed, $p \circ \tau=1$ and $p$ is full, then there exist $\tau(f) \in \operatorname{Hom}_{\mathcal{T}_{1}}(A, B)$ such that $p(\tau(f))=f$ for $f \in$ mor $\mathcal{C}$. Observe that $p(\tau(f g))=$ $f g=p(\tau(f) \circ \tau(g))$, then there exist a 2-morphism $H$ between $\tau f \circ \tau g$ and $\tau(f g)$; see Definition 3.4(i).

We here define the universal Toda bracket.

Hypothesis $I$. Let $\mathcal{G}$ be a track category which satisfies the following conditions:

(i) The group $\mathcal{A}_{X Y}$ is Abelian for any $X$ and $Y$ in $H \mathcal{G}$.

(ii) For the functor $D^{\mathcal{A}}: \mathcal{F}(H \mathcal{G}) \rightarrow A b$ defined in Definition 2.3, there exists a linear track extension

$$
D^{\mathcal{A}} \stackrel{+}{\longrightarrow} \mathcal{G}_{2} \Longrightarrow \mathcal{G}_{1} \stackrel{p}{\longrightarrow} H \mathcal{G}
$$

such that $\sigma_{0}=i d$, where the functor $p: \mathcal{G}_{1} \rightarrow H \mathcal{G}$ is the natural projection.

We observe that $\sigma_{0}$ denotes the element determined by the zero map 0 in the action $\sigma=\left\{\sigma_{f}: D_{[f]}^{\mathcal{A}} \rightarrow \mathcal{G}(f, f) \mid \sigma_{f}\right.$ is an isomorphism of groups for $\left.f \in \operatorname{mor}\left(\mathcal{G}_{1}\right)\right\}$ of $D^{\mathcal{A}}$ which defines the linear track extension above; see [7, proof of Theorem 3.1], [8, Example 4.7] and Example 5.2 below for examples which satisfy Hypothesis I. In particular, Example 5.2 gives a linear track extension whose action consists of identities; that is $\sigma_{f}=i d$ for each morphism $f$ in $\mathcal{G}$.

Definition 3.6. Let $\mathcal{G}$ be a track category which satisfies Hypothesis I and $H \mathcal{G}$ small category. Let $\mathcal{E}(H \mathcal{G})$ be the linear track extension in Hypothesis I. Then the class $\langle H \mathcal{G}\rangle:=\left[C_{\mathcal{E}(H \mathcal{G})}(\tau, H)\right]$ which belongs to $H^{3}\left(H \mathcal{G}, D^{\mathcal{A}}\right)$ is called the universal Toda bracket.

\section{Proof of Theorem 2.6}

We begin by recalling a normalized version of the Baues-Wirsching cohomology. Let $S \subset \operatorname{mor}(\mathcal{C})$ be a subclass of morphism in $\mathcal{C}$. We say that $S$ is an ideal in $\mathcal{C}$ if 
$f \circ g \in S, g \circ h \in S$ for any $g \in S$ and $(f, g, h) \in N_{3}(\mathcal{C})$. A natural system $D$ on $\mathcal{C}$ is $S$-trivial if $f^{*}=0, f_{*}=0$ (zero map) for any $f \in S$. For a 0 -object $*$, a set $O(\mathcal{C})$ is defined by $O(\mathcal{C}):=\{0: A \rightarrow * \rightarrow B \mid A, B \in \mathrm{ob}(\mathcal{C})\}$. It is readily seen that $O(\mathcal{C})$ is an ideal.

Definition 4.1. Let $S$ be an ideal of $\operatorname{mor}(\mathcal{C})$. Abelian subgroups $F^{n}(S)$ and $\check{F}^{n}(S)$ of $F^{n}(\mathcal{C}, D)=F^{n}$ are denoted by

$$
F^{n}(S):= \begin{cases}\left\{c \in F^{n} \mid c\left(\lambda_{1}, \ldots, \lambda_{n}\right)=0 \text { if } \lambda_{i} \in S \text { for every } i \in\{1, \ldots, n\}\right\} & (n \geqslant 1) \\ \left\{c \in F^{0} \mid c(A)=0 \text { if } 1_{A} \in S\right\} & (n=0)\end{cases}
$$

and

$$
\check{F}^{n}(S):= \begin{cases}\left\{c \in F^{n} \mid c\left(\lambda_{1}, \ldots, \lambda_{n}\right)=0 \text { if } \lambda_{i} \in S \text { for some } i \in\{1, \ldots, n\}\right\} & (n \geqslant 1) \\ \left\{c \in F^{0} \mid c(A)=0 \text { if } 1_{A} \in S\right\} & (n=0)\end{cases}
$$

respectively. We say that $c \in F^{n}(S)$ is a cochain relative to $S$. We say that $c \in \check{F}^{n}(S)$ is a normalized cochain.

Observe that we have a sequence $\check{F}^{n}(S) \subset F^{n}(S) \subset F^{n}(\mathcal{C}, D)$ of inclusions.

Let $K$ be a subcategory of a small category $\mathcal{C}$ and $S$ an ideal of $\operatorname{mor}(\mathcal{C})$. We define $H_{S}^{n}(\mathcal{C}, K ; D)$ by $H^{n}\left(F^{*}(\operatorname{mor} K) \cap \check{F}^{*}(S), \delta\right)$, which is called the $S$-normalized cohomology group of the pair $(\mathcal{C}, K)$.

In order to define the map $\alpha$ in Theorem 2.6, important results in $[8]$ concerning the normalized cohomology are described below.

Theorem 4.2 ([8, Theorem (1.9)]). Let $S$ be an ideal in $\mathcal{C}$ and $D$ an $S$-trivial natural system on $\mathcal{C}$. Then the inclusion $j: S \subset S \cup O b(\mathcal{C})$ induces an isomorphism $j^{*}: H_{S \cup O b(\mathcal{C})}^{n}(\mathcal{C}, K ; D) \stackrel{\cong}{\longrightarrow} H_{S}^{n}(\mathcal{C}, K ; D)$ for $n \geqslant 0$.

Theorem 4.3 ([8, Theorem (1.10)]). Let $\mathcal{C}$ be a small category which has a zero object and $O(\mathcal{C})$ the ideal of zero morphisms. Let $K$ be a subcategory of $\mathcal{C}$ which contains the zero morphism 0: $A \rightarrow A$ for every object $A$ in Ob(K). Moreover, let $S$ be an ideal in $\mathcal{C}$ and $D$ a natural system on $\mathcal{C}$ which is $S \cup O(\mathcal{C})$-trivial. Then the inclusion $i: S \subset S \cup O(\mathcal{C})$ induces an isomorphism $i^{*}: H_{S \cup O(\mathcal{C})}^{n}(\mathcal{C}, K ; D) \stackrel{\cong}{\longrightarrow} H_{S}^{n}(\mathcal{C}, K ; D)$.

In what follows, we drop the tildes for objects and morphisms of $M T$ and $S$ denotes the ideal $\{f w, g w, b g w\}$ in $M T$. We shall have a commutative diagram

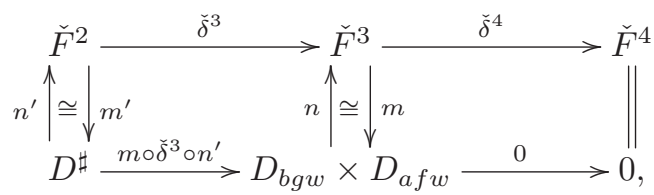

where $D^{\sharp}:=D_{g w} \times D_{b g} \times D_{f w} \times D_{a f} \times D_{b g w}$ and $\check{F}^{i}$ denotes $\check{F}^{i}(S \cup \operatorname{ob}(M T))$; see Appendix A for the commutativity of the diagram and the maps. This allows us to deduce the following lemma.

Lemma 4.4. The map $n$ induces an isomorphism

$$
\tilde{n}: D_{b g w} \times D_{b g w} / I=D_{b g w} \times D_{a f w} / I \cong H_{S \cup \operatorname{ob}(M T)}^{3}(M T, D),
$$

where $I=\operatorname{Im}\left(m \circ \check{\delta}^{3} \circ n^{\prime}\right)$ 
The following lemma is proved in Appendix A.

Lemma 4.5. The map $k: D_{b g w} /\left(b_{*} D_{g w}+w^{*} D_{b g}+a_{*} D_{f w}\right) \rightarrow D_{b g w} \times D_{b g w} / \operatorname{Im}(m \circ$ $\left.\check{\delta}^{3} \circ n^{\prime}\right)$ defined by $k([y])=[(y, 0)]$ is a well-defined isomorphism.

Thus, we have the following result.

Theorem 4.6. Let $D$ be a natural system on $M T$ which is S-trivial. Then $\tilde{n} \circ k$ is an isomorphism from $D_{b g w} /\left(b_{*} D_{g w}+w^{*} D_{b g}+a_{*} D_{f w}\right)$ to $H_{S \cup \operatorname{ob}(M T)}^{3}(M T, D)$.

Let $M T$ be the matrix Toda category, $D^{\mathcal{A}}$ the natural system of automorphism on $H \mathcal{C}$ and $S$ an ideal of $M T$ and of the form $\{f w, g w, b g w\}$. Suppose that there exists a functor $\varphi: M T \rightarrow H \mathcal{G}$ which satisfies the condition that $\varphi(f w)=0$ and $\varphi(g w)=0$. We define $\left(\varphi^{\sharp}\right)^{3}: \check{F}^{3}(\mathrm{O}(H \mathcal{G})) \rightarrow \check{F}^{3}(S)$ by

$$
\left(\varphi^{\sharp}\right)^{3}(c)\left(\lambda_{1}, \lambda_{2}, \lambda_{3}\right)=c\left(\varphi\left(\lambda_{1}\right), \varphi\left(\lambda_{2}\right), \varphi\left(\lambda_{3}\right)\right) .
$$

Moreover, we define $\varphi^{*}: H_{O(H \mathcal{G})}^{3}\left(H \mathcal{G} ; D^{\mathcal{A}}\right) \rightarrow H_{S}^{3}\left(M T ; \varphi^{*} D^{\mathcal{A}}\right)$ by $\varphi^{*}([c]):=\left[\left(\varphi^{\sharp}\right)^{3}(c)\right]$ for $[c] \in H_{O(H \mathcal{G})}^{3}\left(H \mathcal{G} ; D^{\mathcal{A}}\right)$.

We define $\left\{\begin{array}{ll}b & g \\ a & f\end{array}, w\right\}^{*}$ by the composite which fits into the commutative diagram.

$$
\begin{gathered}
H_{O(H \mathcal{G})}^{3}\left(H \mathcal{G} ; D^{\mathcal{A}}\right) \stackrel{\varphi^{*}}{\longrightarrow} H_{S}^{3}\left(M T ; \varphi^{*} D^{\mathcal{A}}\right) \underset{j^{*}}{\stackrel{\cong}{\rightleftarrows}} H_{S \cup \operatorname{ob}(M T)}^{3}\left(M T ; \varphi^{*} D^{\mathcal{A}}\right) \\
\tilde{h} \uparrow \cong \quad \tilde{n} \circ\} \mid \cong \\
H^{3}\left(H \mathcal{G} ; D^{\mathcal{A}}\right) \longrightarrow \mathcal{A}_{W X} / b * \mathcal{A}_{W B}+w^{*} \mathcal{A}_{C X}+a_{*} \mathcal{A}_{W A},
\end{gathered}
$$

where $\tilde{h}$ is the inverse of the isomorphism described in Theorem 4.3; see also Theorem 4.6 .

In order to prove Theorem 2.6, we recall the definition of $\tilde{h}$ in [8, Appendix B]. Under the same assumption as in theorem 4.3 , let $F^{*}$ denote the cochain complex $\left\{F^{n}(\operatorname{mor} K) \cap \check{F}^{n}(S), \delta^{n}\right\}$. We write $F^{n}$ for the module $F^{n}(\operatorname{mor} K) \cap \check{F}^{n}(S)$.

For $n \geqslant 1$ and $0 \leqslant i \leqslant n$, let $t^{i}: F^{n+1} \rightarrow F^{n}$ be a homomorphism defined by

$$
t^{i} c\left(\lambda_{1}, \ldots, \lambda_{n}\right):=\left\{\begin{array}{l}
c\left(\lambda_{1}, \ldots, \lambda_{i}, 0, \lambda_{i+1}, \ldots, \lambda_{n}\right) \text { if } \lambda_{1} \circ \cdots \circ \lambda_{n}=0 \\
0 \quad \text { otherwise. }
\end{array}\right.
$$

By definition, we see that for $n=0, t^{0}: F^{1} \rightarrow F^{0}$ is the trivial map. For each nonnegative integer $k$, define a submodule $F_{k}^{n}$ of $F^{n}$ by

$$
F_{k}^{n}=\left\{c \in F^{n}: c\left(\lambda_{1}, \ldots, \lambda_{n}\right)=0 \text { if } \lambda_{i}=0 \text { for some } i \leqslant k\right\} .
$$

Then $F_{k}^{n}$ defines a decreasing sequence of subcomplexes of $\left(F^{*}, \delta\right)$ such that $F_{0}^{n}=F^{n}$ and $F_{k}^{n}=F^{n}(\operatorname{mor} K) \cap \check{F}^{n}(S \cup O(\mathcal{C}))$ for $k \geqslant n$. We define a map $h^{k}: F_{k}^{*} \rightarrow F_{k}^{*}$ by $h^{k}:=1-\tilde{t^{k}} \delta^{n+1}-\delta^{n} \tilde{t^{k}}$, where

$$
\tilde{t^{k}}(c):=\left\{\begin{array}{l}
(-1)^{k+1} t^{k} \text { if } k \leqslant n-1 \\
0 \quad \text { otherwise. }
\end{array} \text { for } c \in F^{n}\right.
$$

A direct calculation shows that $h^{k}$ is a chain map; see [8, page 337]. Thus we see that $h^{k}$ is homotopic to the identity. The homomorphism $h^{k}$ satisfies the condition that 
(i) $h^{k} c=c$ for $c \in F_{k+1}^{*}$ and (ii) $h^{k}\left(F_{k}^{*}\right) \subset F_{k+1}^{*}$. It follows from the condition (ii) that $h^{k}$ is a homomorphism from $F_{k}^{n}$ to $F_{k+1}^{n}$. Thus we have a commutative diagram

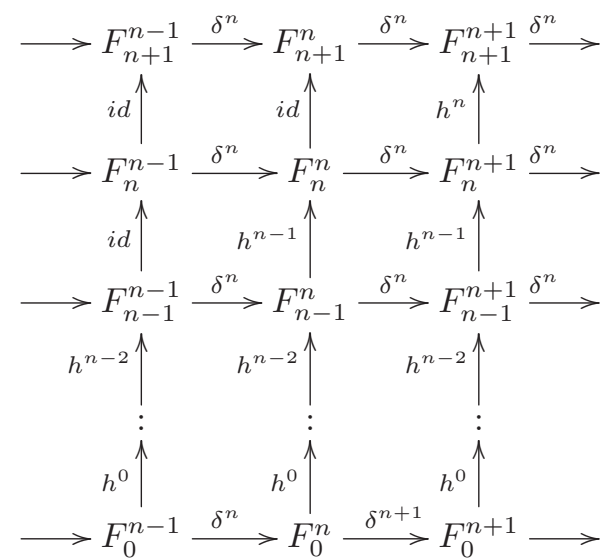

This diagram enables us to define a chain map $h: F_{0}^{n}=F^{n} \rightarrow F_{n}^{n}=F^{n}(\operatorname{mor} K) \cap$ $\check{F}^{n}(S \cup O(\mathcal{C}))$ by $h=h^{n-1} \circ h^{n-2} \circ \cdots \circ h^{0}$. The homomorphism $\tilde{h}$ in Theorem 2.6 is defined by $\tilde{h}([c]):=[h c]$.

We describe Lemma 4.7 which is necessary for Lemma 4.8 .

Lemma 4.7. Suppose that $S$ : af $\Rightarrow$ bg is an invertible 2-morphism in $\mathcal{G}$. If $N(S)$ is a non-empty set, then there exist two invertible 2-morphisms $U:$ af $\Rightarrow 0$ and $V: b g \Rightarrow 0$ such that $S=-V+U$.

Proof. Since $N(S) \neq 0$, there exists 2 -morphism $G: 0 \Rightarrow 0$ such that $G\langle\operatorname{conj}\rangle S$; that is, there exist invertible 2-morphisms $F_{1}$ and $F_{2}$ such that $F_{1}+G=S+F_{2}$. Then we have an equality $S=F_{1}+G-F_{2}$. We define invertible 2-morphisms $U$ : af $\Rightarrow 0$ and $V: b g \Rightarrow 0$ by $V=-G-F_{1}$ and $U=-F_{2}$, respectively. Then we see that $S=$ $-V+U: a f \Rightarrow b g$.

With two important lemmas below, we prove Theorem 2.6; see Appendix B for the proofs of the lemmas 4.8, 4.9.

Lemma 4.8. For the map $k$ in Lemma 4.5, one has $k([\theta])=[(-b K-V w,-a H-$ $U w)]$, where $V$ and $U$ are the 2-morphisms defined by the 2-morphism $S:$ af $\Rightarrow$ bg in Lemma 4.7.

Lemma 4.9. For the composite $h=h^{2} \circ h^{1} \circ h^{0}$, one has

$$
\left(\varphi^{\sharp}\right)^{3}\left(h \mathcal{C}_{\mathcal{E}(C)}(\tau, H)\right)(b, g, w)=\mathcal{C}_{\mathcal{E}(C)}(\tau, H)(b, g, w)=-b K-V w .
$$

Proof of Theorem 2.6. We define a homomorphism

$$
\alpha: H_{S}^{3}\left(M T, \varphi^{*} D^{\mathcal{A}}\right) \stackrel{\cong}{\longrightarrow} \mathcal{A}_{W X} /\left(b_{*} \mathcal{A}_{W B}+w^{*} \mathcal{A}_{C X}+a_{*} \mathcal{A}_{W A}\right)
$$

by $\alpha:=\left(j^{*} \circ \tilde{n} \circ k\right)^{-1}$. 
It follows from Lemma 4.8, 4.4, Theorem 4.2 and Lemma A.1 that

$$
\begin{aligned}
\alpha^{-1}([\theta]) & =j^{*} \circ \tilde{n} \circ k([\theta]) \\
& =\left(j^{*} \circ \tilde{n}\right)[(-b K-V w,-a H-U w)] \\
& =j^{*}[n(-b K-V w,-a H-U w)]=[n(-b K-V w,-a H-U w)] \\
& =\left[c^{\prime}(-b K-V w,-a H-U w)\right]
\end{aligned}
$$

in $H_{S}^{3}\left(M T ; \varphi^{*} D^{\mathcal{A}}\right)$. For $\left[c^{\prime}(-b K-V w,-a H-U w)\right] \in H_{S}^{3}\left(M T ; \varphi^{*} D^{\mathcal{A}}\right)$, we have

$$
c^{\prime}(-b K-V w,-a H-U w)(b, g, w)=-b K-V w .
$$

The same argument enables us to deduce that $c^{\prime}(-b K-V w,-a H-U w)(a, f, w)=-a H-$ $U w$. The result above and Lemma 4.9 yield that $\varphi^{*} \circ \tilde{h}(\langle\mathcal{C}\rangle)=\alpha^{-1}([\theta])$. This completes the proof.

\section{Proof of Theorem 2.14}

In this section, we describe a matrix Toda bracket in a triangulated category in terms of a classical Toda bracket defined in [16, Chapter II].

Let $\mathcal{T}$ be a triangulated category with suspension functor $\Sigma$. We recall here the Toda bracket in $\mathcal{T}$ defined by Heller [16]. Given a diagram $W \stackrel{f}{\rightarrow} X \stackrel{g}{\rightarrow} Y \stackrel{h}{\rightarrow} Z$ with $h g=0$ and $g f=0$ in $\mathcal{T}$. Then we have a distinguished triangle of the form $W \stackrel{f}{\rightarrow}$ $X \stackrel{i}{\rightarrow} C_{f} \stackrel{j}{\rightarrow} \Sigma W$ and a commutative diagram

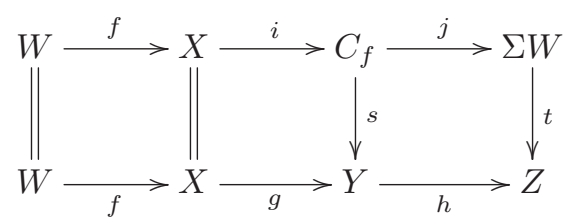

in $\mathcal{T}$. The Toda bracket $\{h, g, f\} \in \mathcal{T}(\Sigma W, Z) /(h \mathcal{T}(\Sigma W, Y)+\mathcal{T}(\Sigma X, Z) \Sigma f)$ is defined by the class $[t]$ in the quotient.

Let $\mathcal{B}$ be an additive category and $C h(\mathcal{B})$ the category of cochain complexes on $\mathcal{B}$ mentioned in Section 2. The homotopy category $H(C h(\mathcal{B}))$, which admits the triangulated category structure whose distinguished triangles are constructed by the mapping cone and suspension functors. Observe that the suspension functor $\Sigma: H(C h(\mathcal{B})) \rightarrow H(C h(\mathcal{B}))$ is defined by $(\Sigma X)^{i}=X^{i+1}$ and $d_{\Sigma X}=-d_{X}$ for a cochain complex $\left(X, d_{X}\right)$.

Let $\mathcal{T}$ be an algebraic triangulated category with a fully faithful exact functor $\vartheta: \mathcal{T} \rightarrow H(C h(\mathcal{B}))$. In what follows, we identify morphisms in $\mathcal{T}$ with their images by $\vartheta$ in $H(C h(\mathcal{B}))$.

Proposition 5.1. $\mathcal{A}_{W X}=\mathcal{A}(0: W \rightarrow X)=\mathcal{T}(\Sigma W, X)$.

Proof. By definition, we see that

$$
\begin{aligned}
\mathcal{A}(0: W \rightarrow X) & =N\left(1_{0}: 0_{X}^{W} \Rightarrow 0_{X}^{W}\right)=\operatorname{Hom}_{\mathcal{T}(W, X)}\left(0_{X}^{W}, 0_{X}^{W}\right) \\
& =\operatorname{Hom}_{\operatorname{ch}(\mathcal{B})(W, X)}\left(0_{X}^{W}, 0_{X}^{W}\right)
\end{aligned}
$$




$$
\begin{aligned}
& =\left\{h: W \rightarrow X \mid 0-0=d_{X} h+h d_{W}\right\} / \sim \\
& =\{h: \Sigma W \rightarrow X \mid h: \text { a cochain map }\} / \text { chain homotopy relation } \\
& =H(C h(\mathcal{B}))(\Sigma W, X) \\
& =\mathcal{T}(\Sigma W, X) .
\end{aligned}
$$

This completes the proof.

We denote by $M D$ the diagram (2.1) in $\mathcal{T}$. Then $M D$ gives rise to a sequence $T(M D): W \stackrel{w}{\rightarrow} C \stackrel{-f \vee g}{\rightarrow} A \oplus B \stackrel{\nabla(a, b)}{\rightarrow} X$ with $(-f \vee g) w=0$ and $\nabla(a, b)(-f \vee g)=0$. Thus $T(M D)$ is regarded as a Toda category by definition.

Proof of Theorem 2.14. We see that the both sides are in the same Abelian group. In fact, the natural maps $i: A \rightarrow A \oplus B$ and $j: B \rightarrow A \oplus B$ induce an isomorphism $\left(i_{*}, j_{*}\right): \mathcal{T}(\Sigma W, A) \oplus \mathcal{T}(\Sigma W, B) \stackrel{\cong}{T}(\Sigma W, A \oplus B)$. It follows from the universality of the direct sum that the composite $\nabla(a, b)_{*} \circ\left(i_{*}, j_{*}\right)$ is nothing but $\nabla\left(a_{*}, b_{*}\right): \mathcal{T}(\Sigma W$, $A) \oplus \mathcal{T}(\Sigma W, B) \rightarrow \mathcal{T}(\Sigma W, X)$. Thus we have

$$
\nabla(a, b)_{*}(\Sigma W, A \oplus B)=a_{*} \mathcal{T}(\Sigma W, A)+b_{*} \mathcal{T}(\Sigma W, B) .
$$

Indeed, we have

$$
\begin{aligned}
\nabla(a, b)_{*} \mathcal{T}(\Sigma W, A \oplus B) & =\nabla(a, b)_{*}\left(i_{*}, j_{*}\right)(\mathcal{T}(\Sigma W, A) \oplus \mathcal{T}(\Sigma w, B)) \\
& =\nabla\left(a_{*}, b_{*}\right)(\mathcal{T}(\Sigma W, A) \oplus \mathcal{T}(\Sigma w, B)) \\
& =a_{*} \mathcal{T}(\Sigma W, A)+b_{*} \mathcal{T}(\Sigma W, B)
\end{aligned}
$$

The result and Proposition 5.1 enable us to conclude that the matrix Toda bracket and the classical Toda bracket are in the same quotient group.

In order to show the equality of Theorem 2.14, we consider a commutative diagram

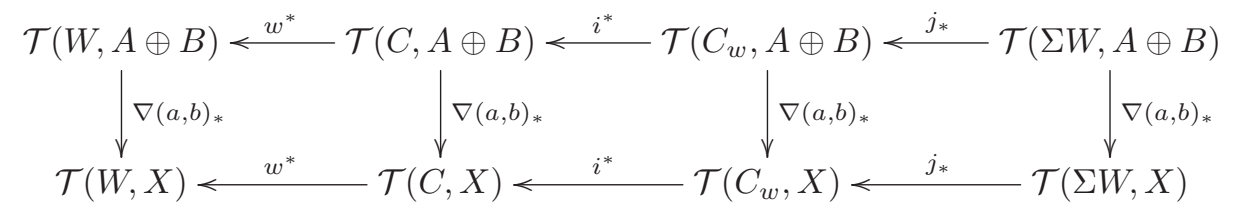

in which row sequences are exact. Chasing the diagram, we construct $t$ and $s$ in the definition of the Toda bracket $\{\nabla(a, b),-f \vee g, w\}$. Since $w^{*}(-f \vee g)=(-f \vee g) w=0$ for $-f \vee g$ in $\mathcal{T}(C, A \oplus B)$, it follows that $i^{*}(s)=-f \vee g$ for some morphism $s: C_{w} \rightarrow$ $A \oplus B$. In fact, we can choose the morphism $\left(\begin{array}{cc}-f & H \\ g & -K\end{array}\right): C_{w}=C \oplus \Sigma W \rightarrow A \oplus$ $B$ in $\mathcal{T}\left(C_{w}, A \oplus B\right)$ as $s$. The equation $C_{w}=C \oplus \Sigma W$ is meant in the underlying category of graded objects in $\mathcal{B}$, not in $\mathcal{T}$. Moreover, we see that $j^{*}(-b K+S w+$ $a H)=(0,-b K+S w+a H), \nabla(a, b)_{*}\left(\begin{array}{cc}-f & H \\ g & -K\end{array}\right)=(-a f+b g, a H-b K)$ and
$j^{*}(-b K+S w+a H)-\nabla(a, b)_{*}\left(\begin{array}{cc}-f & H \\ g & -K\end{array}\right)=(S, 0)\left(\begin{array}{cc}d_{C} & w \\ 0 & -d_{W}\end{array}\right)+d_{X}(S, 0)$.

This implies that we can choose $-b K+S w+a H$ as $t$ and $j_{*}(t)=\nabla(a, b)_{*} s$. This completes the proof. 
We describe a computational example with Theorem 2.14 .

Example 5.2. Let $\mathcal{G}$ be the 2-category $C h(\mathcal{B})$ mentioned above. One has a linear track extension of the form $D^{\mathcal{A}} \stackrel{+}{\longrightarrow} \mathcal{G}_{2} \longrightarrow \mathcal{G}_{1} \stackrel{p}{\longrightarrow} H \mathcal{G}$. By definition, we see that $D_{[f]}^{\mathcal{A}}=\mathcal{A}(0: s(f) \rightarrow t(f))=\mathcal{G}(0,0)=\mathcal{G}(f, f)$. Then, we define the action $\sigma$ by $\sigma_{f}=$ $i d$ for any $f$. Indeed, $\mathcal{G}(f, f)=\left\{h: X \rightarrow Y \mid f-f=d_{Y} h+h d_{X}\right\} / \sim=\{h: X \rightarrow Y \mid$ $\left.0=0-0=d_{Y} h+h d_{X}\right\} / \sim=\mathcal{G}(0,0)$. In this example, all conditions in Theorem 2.6 are satisfied.

Example 5.3. Let $\mathcal{T}$ be the triangulated category $H(C h(\mathcal{B}))$, where $\mathcal{B}$ is the category of $\mathbb{Z}$-modules. Here $\mathcal{T}$ is clearly algebraic. Let $X \stackrel{f}{\rightarrow} Y \stackrel{i}{\rightarrow} C_{f} \stackrel{j}{\rightarrow} \Sigma X$ be the mapping cone construction of a map $f$ in $\mathcal{T}$. Suppose that there exists an integer $k$ such that (i) $H^{k}(X) \neq 0$ while $\operatorname{Hom}\left(H^{k}(Y), H^{k}(X)\right)=0$ and (ii) $H(j): H^{k-1}\left(C_{f}\right) \rightarrow H^{k-1}(\Sigma X)$ is not surjective. Then we see that $1_{\Sigma(X \oplus X)}$ is non-trivial element in the quotient $Q$ defined by

$$
\mathcal{T}\left(\Sigma\left(X^{\oplus 2}\right), \Sigma\left(X^{\oplus 2}\right)\right) /(j, j) \mathcal{T}\left(\Sigma\left(X^{\oplus 2}\right), C_{f}^{\oplus 2}\right)+\mathcal{T}\left(\Sigma\left(Y^{\oplus 2}\right), \Sigma\left(X^{\oplus 2}\right)\right) \Sigma(f, f),
$$

where $A^{\oplus 2}=A \oplus A$. In fact, if $1_{\Sigma(X \oplus X)}$ is trivial in $Q$, then we have

$$
1_{\Sigma(X \oplus X)}=(j, j)(\alpha)+\beta \circ \Sigma(f, f)
$$

for some $\alpha \in H^{0}\left(\operatorname{Hom}\left(\Sigma(X \oplus X), C_{f} \oplus C_{f}\right)\right)$ and $\beta \in H^{0}(\operatorname{Hom}(\Sigma(Y \oplus Y), \Sigma(X \oplus X))$.

Observe that in our setting, the Hom-set $\mathcal{T}(U, V)$ is nothing but the 0th cohomology $H^{0}\left(\operatorname{Hom}\left(\left(U, d_{U}\right),\left(V, d_{V}\right)\right)\right)$ of the cochain complex $\operatorname{Hom}(U, V)$ with the differential $\delta$ defined by $\delta(\varphi)=d_{V} \varphi-(-1)^{\operatorname{deg} \varphi} \varphi d_{U}$ for a homomorphism $\varphi: U \rightarrow V$.

Let $h: H^{0}\left(\operatorname{Hom}\left(\left(U, d_{U}\right),\left(V, d_{V}\right)\right)\right) \rightarrow \prod_{-p+q=0} \operatorname{Hom}\left(H^{p}(U), H^{q}(V)\right)$ be the homomorphism defined by assigning a cochain map the map induced in the cohomology. Then the equality above enables us to deduce that

$$
h\left(1_{\Sigma(X \oplus X)}\right)=(H(j), H(j)) h(\alpha)+h(\beta) \circ \Sigma(H(f), H(f))=(H(j), H(j)) h(\alpha)
$$

in the degree $k-1$. The second equality follows from the condition (i). The left hand side is the identity map $1_{H(\Sigma(X \oplus X))}$ while the right hand side is not surjective because of (ii), which is a contradiction.

We consider a diagram

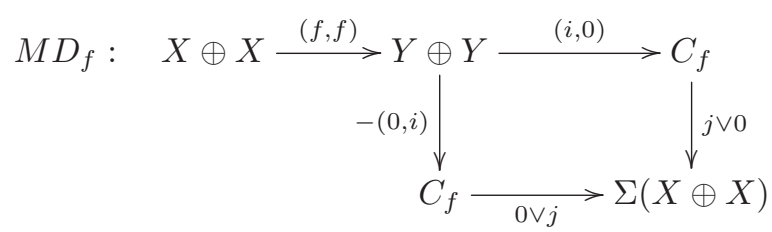

in $\mathcal{T}$. It is readily seen that this is a matrix Toda diagram. Thus the diagram gives rise to a Toda diagram of the form

$$
T\left(M D_{f}\right): \quad X \oplus X \stackrel{(f, f)}{\longrightarrow} Y \oplus Y \stackrel{(i, i)}{\longrightarrow} C_{f} \oplus C_{f} \stackrel{(j, j)}{\longrightarrow} \Sigma(X \oplus X),
$$

which is a distinguished triangle in $\mathcal{T}$. Theorem 2.14 implies that in $Q$,

$$
\left\{\begin{array}{l}
j \vee 0, \\
0 \vee j, \quad-(0, i)
\end{array} \quad(f, f)\right\}=\{(j, j),(i, i),(f, f)\}=\left[1_{\Sigma(X \oplus X)}\right] \neq 0 .
$$


The last equality follows from the definition of the classical Toda bracket.

As a toy example which satisfies the conditions (i) and (ii) above, we give complexes $X, Y$ and a cochain map $f: X \rightarrow Y$, where $X^{0}=\mathbb{Z}, X^{i}=0$ if $i \neq 0, Y^{-1}=$ $Y^{0}=\mathbb{Z}, Y^{k}=0$ for $k \neq-1,0$, the differential $d_{Y}: Y^{-1} \rightarrow Y^{0}$ is defined by $d_{Y}(y)=$ $2 y$ and $f^{0}=1: X^{1}=\mathbb{Z} \rightarrow Y^{1}=\mathbb{Z}$. Observe that $H^{0}(Y)=\mathbb{Z} / 2 \mathbb{Z}, H^{0}(X)=\mathbb{Z}$ and $H(j): H^{-1}\left(C_{f}\right)=2 \mathbb{Z} \rightarrow H^{-1}(\Sigma X)=\mathbb{Z}$ is the inclusion.

The result $[\mathbf{8}$, Theorem (4.6)] asserts that linear track extensions of $\mathcal{C}$ by a natural system $D$ are classified with the Baues-Wirsching cohomology $H^{3}(\mathcal{C}, D)$. Then, Theorem 2.6 enables one to deduce the following result.

Example 5.4. Let $\mathcal{G}$ be a small sub 2-category of $C h(\mathcal{B})$ which contains the matrix Toda diagram $M D_{f}$ described in Example 5.3. There exists a linear track extension of $H \mathcal{G}$ by $D^{\mathcal{A}}$ with $\sigma_{0}=1$ which is non-trivial up to equivalence. This follows from Corollary 2.7.

\section{Appendix A. Proof of Lemma 4.5}

By the same argument as in the proof of [8, Lemma (1.12)], we have Lemma 4.5. We first define isomorphism $m, n, m^{\prime}$ and $n^{\prime}$ in diagram (4.1).

Lemma A.1. The Abelian subgroup $\check{F}^{3}$ of $F^{3}$ is isomorphic to $D_{b g w} \times D_{a f w}$.

Proof. We define homomorphisms $m: \check{F}^{3} \rightarrow D_{b g w} \times D_{a f w}$ and $n: D_{b g w} \times D_{a f w} \rightarrow$ $\check{F}^{3}$ by $m(c):=(c(b, g, w), c(a, f, w))$ and $n\left(\left(x_{1}, x_{2}\right)\right):=c_{\left(x_{1}, x_{2}\right)}^{\prime}$ respectively, where $c_{x_{1}, x_{2}}^{\prime}$ is defined by

$$
c_{\left(x_{1}, x_{2}\right)}^{\prime}\left(\alpha_{1}, \alpha_{2}, \alpha_{3}\right):= \begin{cases}x_{1} & \left(\alpha_{1}, \alpha_{2}, \alpha_{3}\right)=(b, g, w) \\ x_{2} & \left(\alpha_{1}, \alpha_{2}, \alpha_{3}\right)=(a, f, w) \\ 0 & \text { if there exists } i \text { such that } \alpha_{i} \text { is in } S \cup \mathrm{ob}(M T) .\end{cases}
$$

The conditions (ii) and (iii) in the definition of $M T$ yield that the map $n$ is well defined. It is readily seen that $n \circ m=i d_{\check{F}^{3}}$ and $m \circ n=i d_{D_{b g w} \times D_{a f w}}$.

Lemma A.2. The Abelian subgroup $\check{F}^{2}$ of $F^{2}$ is isomorphic to $D^{\sharp}=D_{g w} \times D_{b g} \times$ $D_{f w} \times D_{a f} \times D_{b g w}$.

Proof. We define homomorphisms $m^{\prime}: \check{F}^{2} \rightarrow D^{\sharp}$ and $n^{\prime}: D^{\sharp} \rightarrow \check{F}^{2}$ by

$$
m^{\prime}(c):=(c(g, w), c(b, g), c(f, w), c(a, f),(b g, w))
$$

and

respectively. Here,

$$
n^{\prime}\left(\left(x_{1}, x_{2}, x_{3}, x_{4}, x_{5}\right)\right):=c_{\left(x_{1}, x_{2}, x_{3}, x_{4}, x_{5}\right)}^{\prime},
$$

$$
c_{\left(x_{1}, x_{2}, x_{3}, x_{4}, x_{5}\right)}^{\prime}\left(\alpha_{1}, \alpha_{2}\right):= \begin{cases}x_{1} & \left(\alpha_{1}, \alpha_{2}\right)=(g, w) \\ x_{2} & \left(\alpha_{1}, \alpha_{2}\right)=(b, g) \\ x_{3} & \left(\alpha_{1}, \alpha_{2}\right)=(f, w) \\ x_{4} & \left(\alpha_{1}, \alpha_{2}\right)=(a, f) \\ x_{5} & \left(\alpha_{1}, \alpha_{2}\right)=(b g, w) \\ 0 \text { if there exists } i \text { such that } \alpha_{i} \text { is in } S \cup \operatorname{ob}(M T) .\end{cases}
$$


The well-definedness of $n^{\prime}$ follows from the condition (i) in the definition of $M T$ and the argument of Lemma A.1. A direct calculation shows that $m^{\prime} \circ n^{\prime}=i d_{D^{\sharp}}$ and $n^{\prime} \circ m^{\prime}=i d_{\check{F}^{2}}$.

We show the following lemmas to describe cochains $\check{F}^{4}$ and the map $m \circ \check{\delta}^{3} \circ n^{\prime}$ explicitly.

Lemma A.3. The Abelian subgroup $\check{F}^{n}$ of $F^{n}$ is trivial for $n \geqslant 4$.

Proof. For $\left(\lambda_{1}, \ldots, \lambda_{n}\right) \in N_{n}(M T)$, there exists $i$ such that $\lambda_{i}$ is the identity. Therefore, $c\left(\lambda_{1}, \ldots, \lambda_{n}\right)=0$ for any $c \in \check{F}^{n}$.

Lemma A.4. For $\left(x_{1}, x_{2}, x_{3}, x_{4}, x_{5}\right) \in D^{\sharp}$, one has

$$
m \circ \check{\delta}^{3} \circ n^{\prime}\left(x_{1}, x_{2}, x_{3}, x_{4}, x_{5}\right)=\left(b_{*} x_{1}-w^{*} x_{2}-x_{5}, a_{*} x_{3}-w^{*} x_{4}-x_{5}\right) .
$$

Proof. By definition, it follows that

$$
\begin{aligned}
m \circ \check{\delta}^{3}\left(n ^ { \prime } \left(x_{1}, x_{2}, x_{3},\right.\right. & \left.\left.x_{4}, x_{5}\right)\right) \\
& =m \circ \check{\delta}^{3}\left(c_{\left(x_{1}, x_{2}, x_{3}, x_{4}, x_{5}\right)}^{\prime}\right) \\
& =m\left(\check{\delta}^{3}\left(c_{\left(x_{1}, x_{2}, x_{3}, x_{4}, x_{5}\right)}^{\prime}\right)\right) \\
& =\left(\check{\delta}^{3}\left(c_{\left(x_{1}, x_{2}, x_{3}, x_{4}, x_{5}\right)}^{\prime}\right)(b, g, w), \check{\delta}^{3}\left(c_{\left(x_{1}, x_{2}, x_{3}, x_{4}, x_{5}\right)}^{\prime}\right)(a, f, w)\right) \\
& =\left(\delta^{3}\left(c_{\left(x_{1}, x_{2}, x_{3}, x_{4}, x_{5}\right)}^{\prime}\right)(b, g, w), \delta^{3}\left(c_{\left(x_{1}, x_{2}, x_{3}, x_{4}, x_{5}\right)}^{\prime}\right)(a, f, w)\right) .
\end{aligned}
$$

For the first component, we have

$$
\begin{aligned}
\delta^{3}\left(c_{\left(x_{1}, x_{2}, x_{3}, x_{4}, x_{5}\right)}^{\prime}\right) & (b, g, w) \\
= & b_{*} c_{\left(x_{1}, x_{2}, x_{3}, x_{4}, x_{5}\right)}^{\prime}(g, w)+(-1)^{1} c_{\left(x_{1}, x_{2}, x_{3}, x_{4}, x_{5}\right)}^{\prime}(b g, w) \\
& +(-1)^{2} c_{\left(x_{1}, x_{2}, x_{3}, x_{4}, x_{5}\right)}^{\prime}(b, g w)+(-1)^{3} w^{*}\left(c_{\left(x_{1}, x_{2}, x_{3}, x_{4}, x_{5}\right)}^{\prime}(b, g)\right) \\
= & b_{*} x_{1}-x_{5}+0-w^{*} x_{2}=b_{*} x_{1}-w^{*} x_{2}-x_{5} .
\end{aligned}
$$

For the second one, we see that

$$
\begin{aligned}
\delta^{3}\left(c_{\left(x_{1}, x_{2}, x_{3}, x_{4}, x_{5}\right)}^{\prime}\right) & (a, f, w) \\
= & a_{*} c_{\left(x_{1}, x_{2}, x_{3}, x_{4}, x_{5}\right)}^{\prime}(f, w)+(-1)^{1} c_{\left(x_{1}, x_{2}, x_{3}, x_{4}, x_{5}\right)}^{\prime}(a f, w) \\
& +(-1)^{2} c_{\left(x_{1}, x_{2}, x_{3}, x_{4}, x_{5}\right)}^{\prime}(a, f w)+(-1)^{3} w^{*}\left(c_{\left(x_{1}, x_{2}, x_{3}, x_{4}, x_{5}\right)}^{\prime}(a, f)\right) \\
= & a_{*} x_{3}-x_{5}+0-w^{*} x_{4}=a_{*} x_{3}-w^{*} x_{4}-x_{5} .
\end{aligned}
$$

This completes the proof.

Proof of Lemma 4.5. We define maps

$$
\begin{aligned}
l: D_{b g w} \times D_{b g w} / \operatorname{Im}\left(m \circ \check{\delta}^{3} \circ n^{\prime}\right) & \rightarrow D_{b g w} /\left(b_{*} D_{g w}+w^{*} D_{b g}+a_{*} D_{f w}\right), \\
k: D_{b g w} /\left(b_{*} D_{g w}+w^{*} D_{b g}+a_{*} D_{f w}\right) & \rightarrow D_{b g w} \times D_{b g w} / \operatorname{Im}\left(m \circ \check{\delta}^{3} \circ n^{\prime}\right)
\end{aligned}
$$

by

$$
\begin{aligned}
l\left(\left[\left(x_{1}, x_{2}\right)\right]\right) & :=\left[x_{1}-x_{2}\right] \text { for }\left[\left(x_{1}, x_{2}\right)\right] \in D_{b g w} \times D_{a f w} \operatorname{Im}\left(m \circ \check{\delta}^{3} \circ n^{\prime}\right), \\
k([y]) & :=[(y, 0)] \text { for }[y] \in D_{b g w} /\left(b_{*} D_{g w}+w^{*} D_{b g}+a_{*} D_{f w}\right) .
\end{aligned}
$$

We show that $l$ and $k$ are well-defined homomorphisms. For any element $\left(z_{1}, z_{2}\right)$ in $\operatorname{Im}\left(m \circ \check{\delta}^{3} \circ n^{\prime}\right)$, there exists an element $\left(x_{1}, x_{2}, x_{3}, x_{4}, x_{5}\right) \in D^{\sharp}$ such that $m \circ \check{\delta}^{3} \circ$ $n^{\prime}\left(x_{1}, x_{2}, x_{3}, x_{4}, x_{5}\right)=\left(z_{1}, z_{2}\right)$. By Lemma A.4, we see that $z_{1}=b_{*} x_{1}-w^{*} x_{2}-x_{5}$, 


$$
\begin{aligned}
& z_{2}=a_{*} x_{3}-w^{*} x_{4}-x_{5} \text { and } \\
& \begin{aligned}
z_{1}-z_{2} & =b_{*} x_{1}-w^{*} x_{2}-x_{5}-\left(a_{*} x_{3}-w^{*} x_{4}-x_{5}\right) \\
& =b_{*} x_{1}-a_{*} x_{3}-w^{*}\left(x_{2}-x_{4}\right) \in b_{*} D_{g w}+a_{*} D_{f w}+w^{*} D_{b g} .
\end{aligned}
\end{aligned}
$$

Next, for any element $u$ in $b_{*} D_{g w}+a_{*} D_{f w}+w^{*} D_{b g}$, we write $u=b_{*} x+w^{*} y+$ $a_{*} z$. Then $m \circ \tilde{\delta}^{3} \circ n^{\prime}\left(x,-y,-z, 0,-a_{*} z\right)=(u, 0)$. Observe that $a_{*} z \in a_{*} D_{f w} \subset D_{a f w}$ $=D_{\text {bgw }}$. It is easily seen that $l \circ k=1$ and $k \circ l=1$. Indeed, $l \circ k([y])=l([(y, 0)])=$ $[y-0]=[y]$ and

$$
k \circ l\left(\left[\left(x_{1}, x_{2}\right)\right]\right)=k\left(\left[x_{1}-x_{2}\right]\right)=\left[\left(x_{1}-x_{2}, 0\right)\right]=\left[\left(x_{1}, x_{2}\right)\right] .
$$

In fact, $\left[\left(x_{1}, x_{2}\right)\right]-\left[\left(x_{1}-x_{2}, 0\right)\right]=\left[\left(x_{1}, x_{2}\right)-\left(x_{1}-x_{2}, 0\right)\right]=\left[\left(x_{2}, x_{2}\right)\right]$. Moreover, it follows that $\left(x_{2}, x_{2}\right)$ is in $\operatorname{Im}\left(m \circ \check{\delta}^{3} \circ n^{\prime}\right)$ since $m \circ \check{\delta}^{3} \circ n^{\prime}\left(0,0,0,0,-x_{2}\right)=\left(b_{*} 0-\right.$ $\left.w^{*} 0-\left(-x_{2}\right), a_{*} 0-w^{*} 0-\left(-x_{2}\right)\right)=\left(x_{2}, x_{2}\right)$. We see that $\left[\left(x_{2}, x_{2}\right)\right]=0$ and hence $\left[\left(x_{1}, x_{2}\right)\right]=\left[\left(x_{1}-x_{2}, 0\right)\right]$.

\section{Appendix B. Proofs of Lemmas 4.8 and 4.9}

Explicit calculation gives the proof of the Lemma 4.8 and we describe a proof of Lemma 4.9 in detail.

Proof of Lemma 4.8. Recall that $D^{\mathcal{A}}$ bgw $=\mathcal{A}(0: s($ bgw $) \rightarrow t($ bgw $))=\mathcal{A}(0: W \rightarrow X)$. We see that $U w:$ af $w \Rightarrow 0 w=0: W \rightarrow X$ and then $-U w: 0 \Rightarrow a f w: W \rightarrow X$ and $-a H:$ af $w \Rightarrow 0: W \rightarrow X$. This implies that $-a H-U w: 0 \Rightarrow 0: W \rightarrow X$ and that $(0,0,0,0,-(U w+a H))$ is in $D^{\sharp}=D_{g w}^{\mathcal{A}} \times D_{b g}^{\mathcal{A}} \times D_{f w}^{\mathcal{A}} \times D_{a f}^{\mathcal{A}} \times D^{\mathcal{A}}{ }_{b g w}$. Then we have $[(-b K+(-V w)+U w+a H, 0)]=[(-b K-V w,-a H-U w)]$. In fact, it follows that

$$
\begin{aligned}
(-b K+(-V w)+U & +a H, 0)-(-b K-V w,-a H-U w) \\
= & (-b K+(-V w)+V w+b K+U w+a H, 0-(-a H-U w)) \\
= & (U w+a H, U w+a H) \\
= & m \circ \check{\delta^{3}} \circ n^{\prime}((0,0,0,0,-(U w+a H))) \in \operatorname{Im}\left(m \circ \check{\delta^{3}} \circ n^{\prime}\right) .
\end{aligned}
$$

The last equality follows from Lemma A.4. Therefore, we see that

$$
\begin{aligned}
k([\theta]):=[(\theta, 0)] & =[(-b K+S w+a H, 0)]=[(-b K+(-V+U) w+a H, 0)] \\
& =[(-b K+(-V w)+U w+a H, 0)]=[(-b K-V w,-a H-U w)] .
\end{aligned}
$$

This completes the proof.

Proof of Lemma 4.9. We see that

$$
\mathcal{C}_{\mathcal{E}(C)}(\tau, H)(b, g, w):=\sigma_{\tau(b g w)}^{-1}(\Delta)=\sigma_{\tau(0)}^{-1}(\Delta)=\sigma_{0}^{-1}(\Delta)=\Delta,
$$

since $\sigma_{0}=i d$. We have

$$
\begin{aligned}
\Delta & =-H(b, g w)-(\tau b)_{*} H(g, w)+(\tau w)^{*} H(b, g)+H(b g, w) \\
& =-1_{0_{*}}-(\tau b)_{*} H(g, w)+(\tau w)^{*} H(b, g)+1_{0_{*}} \quad(g w, b g \in S ; \text { see Theorem 4.6) } \\
& =-(\tau b) \circ H(g, w)+H(b, g) \circ(\tau w) \\
& =-b K-V w . \quad(\tau b=b, H(g, w)=K, \tau w=w, H(b, g)=-V)
\end{aligned}
$$

This yields the second equality in Lemma 4.9 . 
Next, we shall show the first equality in Lemma 4.9 . We denote $\mathcal{C}_{\mathcal{E}(C)}(\tau, H)$ by $c$. By the definitions of $\left(\varphi^{\sharp}\right)^{3}$ and the inclusion $\varphi$, we have $\left(\varphi^{\sharp}\right)^{3}(h c)(b, g, w)=h c(\varphi(b), \varphi(g)$, $\varphi(w))=h c(b, g, w)$; see the definition of $\left(\varphi^{\sharp}\right)^{3}$. Since $h=h^{2} \circ h^{1} \circ h^{0}$, it follows that $h c(b, g, w)=\left(h^{2} \circ h^{1} \circ h^{0}\right) c(b, g, w)$, where $h^{0}=\left(1-\tilde{t}^{0} \delta^{4}-\delta^{3} \tilde{t}^{0}\right), h^{1}=\left(1-\tilde{t}^{1} \delta^{4}-\delta^{3} \tilde{t}^{1}\right)$ and $h^{2}=\left(1-\tilde{t}^{2} \delta^{4}-\delta^{3} \tilde{t}^{2}\right)$.

We determine $h^{0} c(b, g, w), h^{1}\left(h^{0} c(b, g, w)\right)$ and $h^{2}\left(h^{1}\left(h^{0} c(b, g, w)\right)\right)$. As for the element $h^{0} c(b, g, w)$, we have

$$
\begin{aligned}
h^{0} c(b, g, w) & =\left(1-\tilde{t}^{0} \delta^{4}-\delta^{3} \tilde{t}^{0}\right) c(b, g, w) \\
& =\left(c-\tilde{t}^{0} \delta^{4}(c)-\delta^{3} \tilde{t}^{0}(c)\right)(b, g, w) \\
& =c(b, g, w)-\delta^{4}(c) \tilde{t}^{0}(b, g, w)-\delta^{3} \tilde{t}^{0}(c)(b, g, w) .
\end{aligned}
$$

Here, $-\delta^{4}(c) \tilde{t}^{0}(b, g, w)$ and $-\delta^{3} \tilde{t}^{0}(c)(b, g, w)$ are determined as follows. By the definitions of $\delta^{4}$ and $\tilde{t}^{k}$, we have

$$
\begin{aligned}
-\delta^{4}(c) \tilde{t}^{0}(b, g, w) & =-\left(\tilde{t}^{0}\left(\delta^{4}(c)\right)(b, g, w)\right) \\
& =-\tilde{t}^{0}\left(\delta^{4}(c)(b, g, w)\right) \\
& =-(-1)^{(0+1)} t^{0}\left(\delta^{4}(c)(b, g, w)\right) \\
& =\delta^{4}(c)(0, b, g, w) \\
& =0_{*} c(b, g, w)-c(0 b, g, w)+c(0, b g, w)-c(0, b, g w)+w^{*} c(0, b, g) \\
& =-c(0, g, w)+c(0, b g, w)-c(0, b, g w)+w^{*} c(0, b, g) .
\end{aligned}
$$

Moreover, we see that

$$
\begin{aligned}
-\delta^{3} \tilde{t}^{0}(c)(b, g, w)= & -\left((-1)^{1} t^{0}(c) b_{*}(g, w)-(-1)^{1} t^{0}(c)(b g, w)\right. \\
& \left.+(-1)^{1} t^{0}(c)(b, g w)-w^{*}(-1)^{1} t^{0}(c)(b, g)\right) \\
= & b_{*} c(0, g, w)-c(0, b g, w)+c(0, b, g w)-w^{*} c(0, b, g) .
\end{aligned}
$$

The definition of $h^{k}$ enables us to calculate $h^{0} c(b, g, w)$ as follows:

$$
\begin{aligned}
h^{0} c(b, g, w)= & c(b, g, w)-\delta^{4}(c) \tilde{t}^{0}(b, g, w)-\delta^{3} \tilde{t}^{0}(c)(b, g, w) \\
= & c(b, g, w)-c(0, g, w)+c(0, b g, w)-c(0, b, g w)+w^{*} c(0, b, g) \\
& +b_{*} c(0, g, w)-c(0, b g, w)+c(0, b, g w)-w^{*} c(0, b, g) \\
= & c(b, g, w)-c(0, g, w)+b_{*} c(0, g, w) .
\end{aligned}
$$

Then, we have

$$
\begin{aligned}
h^{1}\left(h^{0}(b, g, w)\right) & =h^{1}\left(c(b, g, w)-c(0, g, w)+b_{*} c(0, g, w)\right) \\
& =h^{1} c(b, g, w)-h^{1} c(0, g, w)+h^{1} b_{*} c(0, g, w) .
\end{aligned}
$$

Here, we calculate only the first term $h^{1} c(b, g, w)$. The direct calculation shows that

$$
\begin{aligned}
& h^{1} c(b, g, w) \\
&=\left(1-\tilde{t}^{1} \delta^{4}-\delta^{3} \tilde{t}^{1}\right) c(b, g, w) \\
&= c(b, g, w)-\tilde{t}^{1} \delta^{4}(c)(b, g, w)-\delta^{3} \tilde{t}^{1}(c)(b, g, w) \\
&= c(b, g, w)-(-1)^{2} t^{1}\left(\delta^{4}(c)(b, g, w)\right)-\delta^{3}\left((-1)^{2} t^{1}(c)(b, g, w)\right) \\
&= c(b, g, w)-\left(b_{*} c(0, g, w)-c(b 0, g, w)+c(b, 0 g, w)-c(b, 0, g w)+w^{*}(b, 0, g)\right) \\
&-\left(b_{*} t^{1}(c)(g, w)-t^{1}(c)(b g, w)+t^{1}(c)(b, g w)-w^{*} t^{1}(c)(b, g)\right)
\end{aligned}
$$




$$
\begin{aligned}
= & c(b, g, w)-\left(b_{*} c(0, g, w)-c(0, g, w)+c(b, 0, w)-c(b, 0, g w)+w^{*}(b, 0, g)\right) \\
& -\left(b_{*} c(g, 0, w)-c(b g, 0, w)+c(b, 0, g w)-w^{*} c(b, 0, g)\right) \\
= & c(b, g, w)-b_{*} c(0, g, w)+c(0, g, w)-c(b, 0, w)-b_{*} c(g, 0, w)+c(b g, 0, w) .
\end{aligned}
$$

Then, we see that

$$
\begin{aligned}
& h^{1} c(b, g, w)-h^{1} c(0, g, w)+h^{1} b_{*} c(0, g, w) \\
&= c(b, g, w)-b_{*} c(0, g, w)+c(0, g, w)-c(b, 0, w)-b_{*} c(g, 0, w)+c(b g, 0, w) \\
& \quad-\left(c(0, g, w)-0_{*} c(0, g, w)+c(0, g, w)-c(0,0, w)-0_{*} c(g, 0, w)+c(0,0, w)\right) \\
& \quad+b_{*}\left(c(0, g, w)-0_{*} c(0, g, w)+c(0, g, w)-c(0,0, w)-0_{*} c(g, 0, w)+c(0,0, w)\right) \\
&= c(b, g, w)-c(b, 0, w)-b_{*} c(g, 0, w)+c(b g, 0, w)-c(0, g, w)+b_{*} c(0, g, w) .
\end{aligned}
$$

As for the term $h^{2}\left(h^{1}\left(h^{0} c(b, g, w)\right)\right)$, we have

$$
\begin{aligned}
h^{2}( & \left.h^{1}\left(h^{0} c(b, g, w)\right)\right) \\
= & h^{2}\left(c(b, g, w)-c(b, 0, w)-b_{*} c(g, 0, w)+c(b g, 0, w)-c(0, g, w)+b_{*} c(0, g, w)\right) \\
= & h^{2} c(b, g, w)-h^{2} c(b, 0, w)-h^{2} b * c(g, 0, w) \\
& +h^{2} c(b g, 0, w)-h^{2} c(0, g, w)+h^{2} b_{*} c(0, g, w) .
\end{aligned}
$$

As for the first term $h^{2} c(b, g, w)$, it follows that

$$
\begin{aligned}
h^{2} c(b, g, w)= & \left(1-\tilde{t}^{2} \delta^{4}-\delta^{3} \tilde{t}^{2}\right) c(b, g, w) \\
= & c(b, g, w)-(-1)^{3} \delta^{4} t^{2}(c)(b, g, w)-\delta^{3}(-1)^{3} t^{2}(c)(b, g, w) \\
= & c(b, g, w)+\left(b_{*} c(g, 0, w)-c(b g, 0, w)+c(b, 0, w)-c(b, g, 0)+h^{*} c(b, g, 0)\right) \\
& +\left(b_{*} t^{2}(c)(g, w)-t^{2}(c)(b g, w)+t^{2}(c)(b, g w)-h^{*} t^{2}(c)(b, g)\right) \\
= & c(b, g, w)+\left(b_{*} c(g, 0, w)-c(b g, 0, w)+c(b, 0, w)-c(b, g, 0)+h^{*} c(b, g, 0)\right) \\
& +\left(b_{*} c(g, w, 0)-c(b g, w, 0)+c(b, g w, 0)-h^{*} c(b, g, 0)\right) \\
= & c(b, g, w)+b_{*} c(g, 0, w)-c(b g, 0, w)+c(b, 0, w)-c(b, g, 0) \\
& +b_{*} c(g, w, 0)-c(b g, w, 0)+c(b, g w, 0) .
\end{aligned}
$$

Thus, combining the terms with other calculation, we have the following equations:

$$
\begin{aligned}
h^{2}\left(h^{1}(\right. & \left.\left.h^{0} c(b, g, w)\right)\right) \\
= & h^{2} c(b, g, w)-h^{2} c(b, 0, w)-h^{2} b_{*} c(g, 0, w)+h^{2} c(b g, 0, w) \\
& -h^{2} c(0, g, w)+h^{2} b_{*} c(0, g, w) \\
= & c(b, g, w)+b_{*} c(g, 0, w)-c(b g, 0, w)+c(b, 0, w)-c(b, g, 0)+b_{*} c(g, w, 0) \\
& -c(b g, w, 0)+c(b, g w, 0)-\left(c(b, 0, w)+b_{*} c(0,0, w)-c(0,0, w)+c(b, 0, w)\right. \\
& \left.-c(b, 0,0)+b_{*} c(0, w, 0)-c(0, w, 0)+c(b, 0,0)\right)-b_{*}\left(c(g, 0, w)+g_{*} c(0,0, w)\right. \\
& \left.-c(0,0, w)+c(g, 0, w)-c(g, 0,0)+g_{*} c(0, w, 0)-c(0, w, 0)+c(g, 0,0)\right) \\
& +\left(c(b g, 0, w)+(b g)_{*} c(0,0, w)-c(0,0, w)+c(b g, 0, w)-c(b g, 0,0)\right. \\
& \left.+(b g)_{*} c(0, w, 0)-c(0, w, 0)+c(b g, 0,0)\right)-(c(0, g, w)+0 * c(g, 0, w) \\
& \left.-c(0,0, w)+c(0,0, w)-c(0, g, 0)+0_{*} c(g, w, 0)-c(0, w, 0)+c(0, g w, 0)\right) \\
& +b_{*}\left(c(0, g, w)+00_{*} c(g, 0, w)-c(0,0, w)+c(0,0, w)\right. \\
& \left.-c(0, g, 0)+0_{*} c(g, w, 0)-c(0, w, 0)+c(0, g w, 0)\right)
\end{aligned}
$$




$$
\begin{aligned}
= & c(b, g, w)-c(b, g, 0)-c(b g, w, 0)+c(b, g w, 0)-c(b, 0, w)+c(b g, 0, w) \\
& -c(0, g, w)+c(0, g, 0)+c(0, w, 0)-c(0, g w, 0)+b_{*}(c(g, w, 0)-c(g, 0, w) \\
& +c(0, g, w)-c(0, g, 0)-c(0, w, 0)+c(0, g w, 0)) \\
= & c(b, g, w)-c(b, g, 0)-c(0, w, 0)+c(b, 0,0)-c(b, 0, w)+c(0,0, w) \\
& -c(0, g, w)+c(0, g, 0)+c(0, w, 0)-c(0,0,0)+b_{*}(c(g, w, 0)-c(g, 0, w) \\
& +c(0, g, w)-c(0, g, 0)-c(0, w, 0)+c(0,0,0)) .
\end{aligned}
$$

Here, considering the first terms and second one, we have

$$
\begin{aligned}
c(b, g, w) & =\mathcal{C}_{\mathcal{E}(C)}(\tau, H)(b, g, w):=\sigma_{\tau(b g w)}^{-1}(\Delta)=\sigma_{\tau(0)}^{-1}(\Delta)=\Delta \\
& =-H(b, g w)-(\tau b)_{*} H(g, w)+(\tau w)^{*} H(b, g)+H(b g, w) \\
& =-i d_{0_{*}}-(\tau b)_{*} H(g, w)+(\tau w)^{*} H(b, g)+i d_{0_{*}} \\
& =-(\tau b)_{*} H(g, w)+(\tau w)^{*} H(b, g) \\
& =-(\tau b) \circ H(g, w)+H(b, g) \circ(\tau w), \\
c(b, g, 0) & =-H(b, 0)-(\tau b)_{*} H(g, 0)+(\tau 0)^{*} H(b, g)+H(b g, 0) \\
& =-i d-(\tau b)_{*} H(g, 0)+(0)^{*} H(b, g)+i d \\
& =-(\tau b)_{*} i d_{\alpha}+0^{*} H(b, g) \\
& =-i d_{(\tau b) \circ \alpha}+0^{*} H(b, g) \\
& =H(b, g) \circ 0_{*} .
\end{aligned}
$$

In consequence, we see that

$$
\begin{aligned}
& h^{2}\left(h^{1}\left(h^{0} c(b, g, w)\right)\right) \\
&= c(b, g, w)-H(b, g) \circ 0_{*}+H(b, 0) \circ 0_{*} \\
&-(-(\tau b) \circ H(0, w)+H(b, 0) \circ(\tau w))+(-0 \circ H(0, w)+H(0,0) \circ(\tau w)) \\
&-(-0 \circ H(g, w)+H(0, g) \circ(\tau w))+H(0, g) \circ 0_{*}-H(0,0) \circ 0_{*} \\
&+b_{*}\left[H(g, w) \circ 0_{*}-\{(-\tau g) \circ H(0, w)+H(g, 0) \circ(\tau w)\}-0 \circ H(g, w)\right. \\
&\left.+H(0, g) \circ(\tau w)-H(0, g) \circ 0_{*}-H(0, w) \circ 0_{*}+H(0,0) \circ 0_{*}\right] .
\end{aligned}
$$

In the equation above, observe that

$$
\begin{aligned}
H(b, 0) \circ(\tau w) & =i d \circ \tau w=i d_{\alpha} \circ i d_{\tau w}=i d_{\alpha(\tau w)}=i d_{0}, \\
\tau g \circ H(0, w)-H(g, 0) \circ(\tau w)+H(0, g) \circ(\tau w) & =(\tau g) \circ i d-i d \circ(\tau w)+i d \circ(\tau w) \\
& =i d_{\tau g} \circ i d=i d_{(\tau g) \circ \alpha}=i d_{0}, \\
-H(0, g) \circ 0_{*}+H(0,0) \circ 0_{*} & =-i d \circ 0_{*}+i d \circ 0_{*}, \\
b_{*}\left(i d_{0}\right) & =i d_{b 0}=i d_{0}, \\
-b_{*} \circ\left(H(0, w) \circ 0_{*}\right) & =-b_{*} \circ\left(i d \circ 0_{*}\right)=-b_{*} \circ i d_{0} \\
& =-(\tau b) \circ H(0, w) .
\end{aligned}
$$

Then, we have

$$
\begin{aligned}
h^{2}\left(h^{1}\left(h^{0} c(b, g, w)\right)\right)= & c(b, g, w)-H(b, 0) \circ(\tau w)+H(0,0) \circ(\tau w) \\
& -H(0, g) \circ(\tau w)+b_{*} \circ i d_{0} \\
= & c(b, g, w)-i d_{0}+i d \circ \tau w-i d \circ \tau w+i d_{0}
\end{aligned}
$$




$$
\begin{aligned}
& =c(b, g, w) \\
& =\mathcal{C}_{\mathcal{E}(C)}(\tau, H)(b, g, w) \\
& =-b K-V w .
\end{aligned}
$$

In the same argument as above enables us to conclude that $\mathcal{C}_{\mathcal{E}(C)}(\tau, H)(a, f, w)=$ $-a H-U w$. This completes the proof.

\section{References}

[1] M. Banagl, Topological Invariants of Stratified Spaces. Springer Monogr. Math. Springer, Berlin, 2007.

[2] M.G. Barratt, Homotopy operations and homotopy groups, 1963. A.M.S. Summer Topology Institute (Seattle).

[3] H.J. Baues, D. Blanc, and S. Gondhali, Higher Toda brackets and Massey products. J. Homotopy Relat. Struct., 11:643-677, 2016.

[4] H.J. Baues and M. Jibladze, Classification of Abelian track categories, K-Theory, 25:299-311, 2002.

[5] H.J. Baues and F. Muro, The homotopy category of pseudofunctors and translation cohomology. J. Pure Appl. Algebra, 211:821-850, 2007.

[6] H.J. Baues, Algebraic Homotopy, volume 15 of Cambridge Stud. Adv. Math. Cambridge University Press, Cambridge, 1989.

[7] H.J. Baues, On the cohomology of categories, universal Toda brackets and homotopy pairs. K-Theory, 11(3):259-285, 1997.

[8] H.J. Baues and W. Dreckmann, The cohomology of homotopy categories and the general linear group. K-Theory, 3(4):307-338, 1989.

[9] H.J. Baues and G. Wirsching, Cohomology of small categories. J. Pure Appl. Algebra, 38(2-3):187-211, 1985.

[10] D. Blanc and S. Paoli, Two-track categories. J. K-Theory, 8(1):59-106, 2011.

[11] J.D. Christensen and M. Frankland, Higher Toda brackets and the Adams spectral sequence in triangulated categories. Algebr. Geom. Topol. 17(5):26872735, 2017.

[12] P.G. Goerss and J.F. Jardine, Simplicial Homotopy Theory. Modern Birkhäuser Classics. Birkhäuser Verlag, Basel, 2009. Reprint of the 1999 edition [MR1711612].

[13] K.A. Hardie, K.H. Kamps, and H.J. Marcum, A categorical approach to matrix Toda brackets. Trans. Amer. Math. Soc., 347(12):4625-4649, 1995.

[14] K.A. Hardie, K.H. Kamps, H.J. Marcum, and N. Oda, Triple brackets and lax morphism categories. Appl. Categ. Structures, 12(1):3-27, 2004.

[15] K.A. Hardie, H.J. Marcum, and N. Oda, Bracket operations in the homotopy theory of a 2-category. Rend. Istit. Mat. Univ. Trieste, 33(1-2):19-70 (2002), 2001.

[16] A. Heller, Stable homotopy categories. Bull. Amer. Math. Soc., 74:28-63, 1968. 
[17] K.H. Kamps and T. Porter, Abstract Homotopy and Simple Homotopy Theory. World Scientific Publishing Co., Inc., River Edge, NJ, 1997.

[18] H. Krause, Derived categories, resolutions, and Brown representability. In Interactions between Homotopy Theory and Algebra, volume 436 of Contemp. Math., pages 101-139. Amer. Math. Soc., Providence, RI, 2007.

[19] M. Mimura, On the generalized Hopf homomorphism and the higher composition. I. J. Math. Kyoto Univ., 4:171-190, 1964.

[20] F. Muro, S. Schwede and N. Strickland, Triangulated categories without models. Invent. Math. 170:231-241, 2007.

[21] H. Toda, Composition Methods in Homotopy Groups of Spheres, volume 49 of Ann. of Math. Stud. Princeton University Press, Princeton, NJ, 1962.

Yasuhiro Momose momose@math.shinshu-u.ac.jp

Department of Mathematical Sciences, Shinshu University, 3-1-1 Asahi, Matsumoto, Nagano, 390-8621, Japan

Kenichirou Shinkai 13st204h@shinshu-u.ac.jp

Department of Mathematical Sciences, Shinshu University, 3-1-1 Asahi, Matsumoto, Nagano, 390-8621, Japan 